\title{
SYMMETRIC TENSORS AND SYMMETRIC TENSOR RANK
}

\author{
PIERRE COMON*, GENE GOLUB ${ }^{\dagger}$, LEK-HENG LIM ${ }^{\dagger}$, AND BERNARD MOURRAIN ${ }^{\ddagger}$
}

Abstract. A symmetric tensor is a higher order generalization of a symmetric matrix. In this paper, we study various properties of symmetric tensors in relation to a decomposition into a symmetric sum of outer product of vectors. A rank-1 order- $k$ tensor is the outer product of $k$ non-zero vectors. Any symmetric tensor can be decomposed into a linear combination of rank-1 tensors, each of them being symmetric or not. The rank of a symmetric tensor is the minimal number of rank- 1 tensors that is necessary to reconstruct it. The symmetric rank is obtained when the constituting rank- 1 tensors are imposed to be themselves symmetric. It is shown that rank and symmetric rank are equal in a number of cases, and that they always exist in an algebraically closed field. We will discuss the notion of the generic symmetric rank, which, due to the work of Alexander and Hirschowitz, is now known for any values of dimension and order. We will also show that the set of symmetric tensors of symmetric rank at most $r$ is not closed, unless $r=1$.

Key words. Tensors, multiway arrays, outer product decomposition, symmetric outer product decomposition, CANDECOMP, PARAFAC, tensor rank, symmetric rank, symmetric tensor rank, generic symmetric rank, maximal symmetric rank, quantics

AMS subject classifications. 15A03, 15A21, 15A72, 15A69, 15A18

1. Introduction. We will be interested in the decomposition of a symmetric tensor into a minimal linear combination of symmetric outer products of vectors (i.e. of the form $\mathbf{v} \otimes \mathbf{v} \otimes \cdots \otimes \mathbf{v}$ ). We will see that a decomposition of the form

$$
A=\sum_{i=1}^{r} \lambda_{i} \mathbf{v}_{i} \otimes \mathbf{v}_{i} \otimes \cdots \otimes \mathbf{v}_{i}
$$

always exists for any symmetric tensor $A$ (over any field). One may regard this as a generalization of the eigenvalue decomposition for symmetric matrices to higher order symmetric tensors. In particular, this will allow us to define a notion of symmetric tensor rank (as the minimal $r$ over all such decompositions) that reduces to the matrix rank for order-2 symmetric tensors.

We will call (1.1) the symmetric outer product decomposition of the symmetric tensor $A$ and we will establish its existence in Proposition 4.2. This is often abbreviated as CAND in signal processing. The decomposition of a tensor into an (asymmetric) outer product of vectors and the corresponding notion of tensor rank was first introduced and studied by Frank L. Hitchcock in 1927 [29, 30]. This same decomposition was rediscovered in the 1970s by psychometricians in their attempts to define data analytic models that generalize factor analysis to multiway data [59]. The name CANDECOMP, for 'canonical decomposition', was used by Carrol and Chang 11] while the name PARAFAC, for 'parallel factor analysis', was used by Harshman [28] for their respective models.

The symmetric outer product decomposition is particularly important in the process of blind identification of under-determined mixtures (UDM), i.e. linear mixtures with more inputs than observable outputs. We refer the reader to [14, 17, 20, 49, 50] and references therein for a list of other application areas, including speech, mobile communications, machine learning, factor analysis of $k$-way arrays, biomedical engineering, psychometrics, and chemometrics.

\footnotetext{
${ }^{*}$ Laboratoire I3S, CNRS and the University of Nice, Sophia-Antipolis, France, p.comon@ieee.org

${ }^{\dagger}$ Department of Computer Science and the Institute for Computational and Mathematical Engineering, Stanford University, Stanford, CA, USA, golub, lekheng@cs.stanford.edu

¥Projet GALAAD, INRIA, Sophia-Antipolis, France, mourrain@sophia.inria.fr
} 
Despite a growing interest in the symmetric decomposition of symmetric tensors, this topic has not been adequately addressed in the general literature, and even less so in the engineering literature. For several years, the alternating least squares algorithm has been used to fit data arrays to a multilinear model [36, 50]. Yet, the minimization of this matching error is an ill-posed problem in general, since the set of symmetric tensors of symmetric rank not more than $r$ is not closed, unless $r=1$ (see Sections 6 and 8) - a fact that parallels the illposedness discussed in [21]. The focus of this paper is mainly on symmetric tensors. The asymmetric case will be addressed in a companion paper, and will use similar tools borrowed from algebraic geometry.

Symmetric tensors form a singularly important class of tensors. Examples where these arise include higher order derivatives of smooth functions [40], and moments and cumulants of random vectors 43]. The decomposition of such symmetric tensors into simpler ones, as in the symmetric outer product decomposition, plays an important role in independent component analysis [14] and constitutes a problem of interest in its own right. On the other hand the asymmetric version of the outer product decomposition defined in (4.1) is central to multiway factor analysis 50.

In Sections 2 and 3, we discuss some classical results in multilinear algebra [5, 26, 39, 42, 44, 63, and algebraic geometry [27, 64. While these background materials are well-known to many pure mathematicians, we found that practitioners and applied mathematicians (in signal processing, neuroimaging, numerical analysis, optimization, etc) - for whom this paper is intended - are often unaware of these classical results. For instance, some do not realize that the classical definition of a symmetric tensor given in Definition 3.2 is equivalent to the requirement that the coordinate array representing the tensor be invariant under all permutations of indices, as in Definition 3.1. Many authors have persistently mislabeled the latter a 'supersymmetric tensor' (cf. 10, 34, 45). In fact, we have found that even the classical definition of a symmetric tensor is not as well-known as it should be. We see this as an indication of the need to inform our target readership. It is our hope that the background materials presented in Sections 2 and 3 will serve such a purpose.

Our contributions will only begin in Section 4 , where the notions of maximal and generic rank are analyzed. The concepts of symmetry and genericity are recalled in Sections 3 and 4, respectively. The distinction between symmetric rank and rank is made in Section 1 , and it is shown in Section 5 that they must be equal in specific cases. It is also pointed out in Section 6 that the generic rank always exists in an algebraically closed field, and that it is not maximal except in the binary case. More precisely, the sequence of sets of symmetric tensors of symmetric rank $r$ increases with $r$ (in the sense of inclusion) up to the generic symmetric rank, and decreases thereafter. In addition, the set of symmetric tensors of symmetric rank at most $r$ and order $d>2$ is closed only for $r=1$ and $r=R_{\mathrm{S}}$, the maximal symmetric rank. Values of the generic symmetric rank and the uniqueness of the symmetric outer product decomposition are addressed in Section 6. In Section 8, we give several examples of sequences of symmetric tensors converging to limits having strictly higher symmetric ranks. We also give an explicit example of a symmetric tensor whose values of symmetric rank over $\mathbb{R}$ and over $\mathbb{C}$ are different.

In this paper, we restrict our attention mostly to decompositions over the complex field. A corresponding study over the real field will require techniques rather different from those introduced here, as we will elaborate in Section 8.2 .

2. Arrays and tensors. A $k$-way array of complex numbers will be written in the form $A=\llbracket a_{j_{1} \ldots j_{k}} \rrbracket_{j_{1}, \ldots, j_{k}=1}^{n_{1}, \ldots, n_{k}}$, where $a_{j_{1} \cdots j_{k}} \in \mathbb{C}$ is the $\left(j_{1}, \ldots, j_{k}\right)$-entry of 
the array. This is sometimes also called a $k$-dimensional hypermatrix. We denote the set of all such arrays by $\mathbb{C}^{n_{1} \times \cdots \times n_{k}}$, which is evidently a complex vector space of dimension $n_{1} \cdots n_{k}$ with respect to entry-wise addition and scalar multiplication. When there is no confusion, we will leave out the range of the indices and simply write $A=\llbracket a_{j_{1} \cdots j_{k}} \rrbracket \in \mathbb{C}^{n_{1} \times \cdots \times n_{k}}$.

Unless noted otherwise, arrays with at least two indices will be denoted in uppercase; vectors are one-way arrays, and will be denoted in bold lowercase. For our purpose, only a few notations related to arrays [14, 20] are necessary.

The outer product (or Segre outer product) of $k$ vectors $\mathbf{u} \in \mathbb{C}^{n_{1}}, \mathbf{v} \in \mathbb{C}^{n_{2}}, \ldots, \mathbf{z} \in$ $\mathbb{C}^{n_{k}}$ is defined as

$$
\mathbf{u} \otimes \mathbf{v} \otimes \cdots \otimes \mathbf{z}:=\llbracket u_{j_{1}} v_{j_{2}} \cdots z_{j_{k}} \rrbracket_{j_{1}, j_{2}, \ldots, j_{k}=1}^{n_{1}, n_{2}, \ldots, n_{k}} .
$$

More generally, the outer product of two arrays $A$ and $B$, respectively of orders $k$ and $\ell$, is an array of order $k+\ell, C=A \otimes B$ with entries

$$
c_{i_{1} \cdots i_{k} j_{1} \cdots j_{\ell}}:=a_{i_{1} \cdots i_{k}} b_{j_{1} \cdots j_{\ell}}
$$

For example, the outer product of two vectors, $\mathbf{u} \otimes \mathbf{v}$, is a matrix. The outer product of three vectors, or of a matrix with a vector, is a 3 -way array.

How is an array related to a tensor? Recall that a tensor is simply an element in the tensor product of vector spaces $[5,26,39,42,44,63]$. One may easily check that the so-called Segre map

$$
\begin{aligned}
\varphi: \mathbb{C}^{n_{1}} \times \cdots \times \mathbb{C}^{n_{k}} & \rightarrow \mathbb{C}^{n_{1} \times \cdots \times n_{k}}, \\
(\mathbf{u}, \ldots, \mathbf{z}) & \mapsto \mathbf{u} \otimes \cdots \otimes \mathbf{z}
\end{aligned}
$$

is multilinear. By the universal property of the tensor product [6, 26, 39, 42, 44, 63], there exists a linear map $\theta$

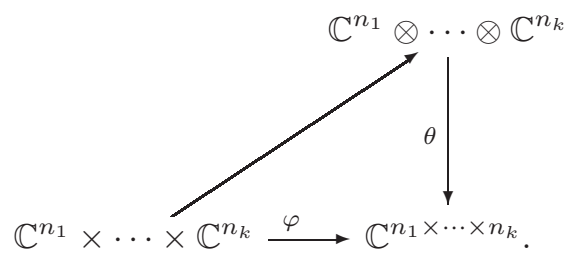

Since $\operatorname{dim}\left(\mathbb{C}^{n_{1}} \otimes \cdots \otimes \mathbb{C}^{n_{k}}\right)=\operatorname{dim}\left(\mathbb{C}^{n_{1} \times \cdots \times n_{k}}\right), \theta$ is an isomorphism of the vector spaces $\mathbb{C}^{n_{1}} \otimes \cdots \otimes \mathbb{C}^{n_{k}}$ and $\mathbb{C}^{n_{1} \times \cdots \times n_{k}}$. Consider the canonical basis of $\mathbb{C}^{n_{1}} \otimes \cdots \otimes \mathbb{C}^{n_{k}}$,

$$
\left\{\mathbf{e}_{j_{1}}^{(1)} \otimes \cdots \otimes \mathbf{e}_{j_{k}}^{(k)} \mid 1 \leq j_{1} \leq n_{1}, \ldots, 1 \leq j_{k} \leq n_{k}\right\},
$$

where $\left\{\mathbf{e}_{1}^{(\ell)}, \ldots, \mathbf{e}_{n_{\ell}}^{(\ell)}\right\}$ denotes the canonical basis in $\mathbb{C}^{n_{\ell}}, \ell=1, \ldots, k$. Then $\theta$ may be described by

$$
\theta\left(\sum_{j_{1}, \ldots, j_{k}=1}^{n_{1}, \ldots, n_{k}} a_{j_{1}, \ldots, j_{k}} \mathbf{e}_{j_{1}}^{(1)} \otimes \cdots \otimes \mathbf{e}_{j_{k}}^{(k)}\right)=\llbracket a_{j_{1} \ldots j_{k}} \rrbracket_{j_{1}, \ldots, j_{k}=1}^{n_{1}, \ldots, n_{k}} .
$$

So an order- $k$ tensor in $\mathbb{C}^{n_{1}} \otimes \cdots \otimes \mathbb{C}^{n_{k}}$ and a $k$-way array in $\mathbb{C}^{n_{1} \times \cdots \times n_{k}}$ that represents the tensor with respect to a basis may be regarded as synonymous (up to, of course, the choice of basis). We will illustrate how the $k$-array representation of an order- $k$ tensor is affected by a change-of-basis. Let $A=\llbracket a_{i j k} \rrbracket \in \mathbb{C}^{n_{1} \times n_{2} \times n_{3}}$ and let $L, M$, 
and $N$ be three matrices of size $r_{1} \times n_{1}, r_{2} \times n_{2}$, and $r_{3} \times n_{3}$, respectively. Then the tensor $A$ may be transformed by the multilinear map $(L, M, N)$ into a tensor $A^{\prime}=\llbracket a_{p q r}^{\prime} \rrbracket \in \mathbb{C}^{r_{1} \times r_{2} \times r_{3}}$ defined by

$$
a_{p q r}^{\prime}=\sum_{i, j, k} l_{p i} m_{q j} n_{r k} a_{i j k} .
$$

When $r_{i}=n_{i}$ and $L, M, N$ are nonsingular matrices, the above multilinear map may be thought of as a change-of-bases (refer to 21] for further discussions). We will call this map a multilinear transform of $A$.

In addition to the outer product, we also have an inner product or contraction product of two arrays. The mode- $p$ inner product between two arrays $A, B$ having the same $p$ th dimension is denoted $A \bullet{ }_{p} B$, and is obtained by summing over the $p$ th index. More precisely, if $A$ and $B$ are of orders $k$ and $\ell$ respectively, this yields for $p=1$ the array $C=A \bullet{ }_{1} B$ of order $k+\ell-2$ :

$$
c_{i_{2} \cdots i_{k} j_{2} \cdots j_{\ell}}=\sum_{\alpha} a_{\alpha i_{2} \cdots i_{k}} b_{\alpha j_{2} \cdots j_{\ell}} .
$$

Note that some authors [20, 24, 59] denoted this contraction product as $A \times{ }_{p} B$ or $\langle A, B\rangle_{p}$. By convention, when the contraction is between a tensor and a matrix, it is convenient to assume that the summation is always done on the second matrix index. For instance, the multilinear transform in 2.1 may be expressed as $A^{\prime}=$ $A \bullet \bullet_{1} L \bullet_{2} M \bullet \bullet_{3} N$. An alternative notation for (2.1) from the theory of group actions is $A^{\prime}=(L, M, N) \cdot A$, which may be viewed as multiplying $A$ on 'three sides' by the matrices $L, M$, and $N$ 21, 32].

3. Symmetric arrays and symmetric tensors. We shall say that a $k$-way array is cubical if all its $k$ dimensions are identical, i.e. $n_{1}=\cdots=n_{k}=n$. A cubical array will be called symmetric if its entries do not change under any permutation of its $k$ indices. Formally, if $\mathfrak{S}_{k}$ denotes the symmetric group of permutations on $\{1, \ldots, k\}$, then we have

DEFINITION 3.1. A $k$-way array $\llbracket a_{j_{1} \cdots j_{k}} \rrbracket \in \mathbb{C}^{n \times \cdots \times n}$ is called symmetric if

$$
a_{i_{\sigma(1)} \cdots i_{\sigma(k)}}=a_{i_{1} \cdots i_{k}}, \quad i_{1}, \ldots, i_{k} \in\{1, \ldots, n\},
$$

for all permutations $\sigma \in \mathfrak{S}_{k}$.

For example, a 3 -way array $\llbracket a_{i j k} \rrbracket \in \mathbb{C}^{n \times n \times n}$ is symmetric if

$$
a_{i j k}=a_{i k j}=a_{j i k}=a_{j k i}=a_{k i j}=a_{k j i}
$$

for all $i, j, k \in\{1, \ldots, n\}$.

Such arrays have been improperly labeled 'supersymmetric' tensors (cf. [10, 34, 45] among others); this terminology should be avoided since it refers to an entirely different class of tensors [7]. The word 'supersymmetric' has always been used in both mathematics and physics $25,60,62$ to describe objects with a $\mathbb{Z}_{2}$-grading and so using it in the sense of [10, 34, 45] is both inconsistent and confusing (the correct usage will be one in the sense of [7]). In fact, we will show below in Proposition 3.7 that there is no difference between Definition 3.1 and the usual definition of a symmetric tensor in mathematics [5, 26, 39, 42, 44, 63]. In other words, the prefix 'super' in 'supersymmetric tensor', when used in the sense of [10, 34, 45], is superfluous.

We will write $\mathrm{T}^{k}\left(\mathbb{C}^{n}\right):=\mathbb{C}^{n} \otimes \cdots \otimes \mathbb{C}^{n}(k$ copies $)$, the set of all order- $k$ dimension$n$ cubical tensors. We define a group action $\mathfrak{S}_{k}$ on $\mathrm{T}^{k}\left(\mathbb{C}^{n}\right)$ as follows. For any $\sigma \in \mathfrak{S}_{k}$ and $\mathbf{x}_{i_{1}} \otimes \cdots \otimes \mathbf{x}_{i_{k}} \in \mathrm{T}^{k}\left(\mathbb{C}^{n}\right)$, we let

$$
\sigma\left(\mathbf{x}_{i_{1}} \otimes \cdots \otimes \mathbf{x}_{i_{k}}\right):=\mathbf{x}_{i_{\sigma(1)}} \otimes \cdots \otimes \mathbf{x}_{i_{\sigma(k)}}
$$


and extend this linearly to all of $\mathrm{T}^{k}\left(\mathbb{C}^{n}\right)$. Thus each $\sigma \in \mathfrak{S}_{k}$ defines a linear operator $\sigma: \mathrm{T}^{k}\left(\mathbb{C}^{n}\right) \rightarrow \mathrm{T}^{k}\left(\mathbb{C}^{n}\right)$. The standard definition of a symmetric tensor in mathematics [5, 26, 39, 42, 44, 63] looks somewhat different from Definition 3.1 and is given as follows.

Definition 3.2. An order- $k$ tensor $A \in \mathrm{T}^{k}\left(\mathbb{C}^{n}\right)$ is symmetric if

$$
\sigma(A)=A
$$

for all permutations $\sigma \in \mathfrak{S}_{k}$. The set of symmetric tensors in $\mathrm{T}^{k}\left(\mathbb{C}^{n}\right)$ will be denoted by $\mathrm{S}^{k}\left(\mathbb{C}^{n}\right)$.

Let $S: \mathrm{T}^{k}\left(\mathbb{C}^{n}\right) \rightarrow \mathrm{T}^{k}\left(\mathbb{C}^{n}\right)$ be the linear operator defined by

$$
S:=\frac{1}{k !} \sum_{\sigma \in \mathfrak{S}_{k}} \sigma .
$$

Note that given any $\sigma \in \mathfrak{S}_{k}$,

$$
\sigma \circ S=S \circ \sigma=S
$$

Here $\circ$ denotes the composition of the linear operators $\sigma$ and $S$.

Proposition 3.3. An order- $k$ tensor $A \in \mathrm{T}^{k}\left(\mathbb{C}^{n}\right)$ is symmetric if and only if

$$
S(A):=\frac{1}{k !} \sum_{\sigma \in \mathfrak{S}_{k}} \sigma(A)=A .
$$

Proof. Clearly, if $A$ is symmetric, then

$$
S(A)=\frac{1}{k !} \sum_{\sigma \in \mathfrak{S}_{k}} \sigma(A)=\frac{1}{k !} \sum_{\sigma \in \mathfrak{S}_{k}} A=A .
$$

Conversely, if $S(A)=A$, then

$$
\sigma(A)=\sigma(S(A))=\sigma \circ S(A)=S(A)=A
$$

for all $\sigma \in \mathfrak{S}_{k}$; and so $A$ is symmetric.

In other words, a symmetric tensor is an eigenvector of the linear operator $S$ with eigenvalue 1. $\mathrm{S}^{k}\left(\mathbb{C}^{n}\right)$ is the 1-eigenspace of $S: \mathrm{T}^{k}\left(\mathbb{C}^{n}\right) \rightarrow \mathrm{T}^{k}\left(\mathbb{C}^{n}\right)$. Proposition 3.3 implies that $\mathrm{S}^{k}\left(\mathbb{C}^{n}\right)=S\left(\mathrm{~T}^{k}\left(\mathbb{C}^{n}\right)\right)$ and it is also easy to see that $S$ is a projection of $\mathrm{T}^{k}\left(\mathbb{C}^{n}\right)$ onto the subspace $\mathrm{S}^{k}\left(\mathbb{C}^{n}\right)$, i.e. $S^{2}=S$.

3.1. Equivalence with homogeneous polynomials. We adopt the following standard shorthand. For any $\mathbf{e}_{i_{1}}, \ldots, \mathbf{e}_{i_{k}} \in \mathbb{C}^{n}$ with $i_{1}, \ldots, i_{k} \in\{1, \ldots, n\}$, we write

$$
\mathbf{e}_{i_{1}} \cdots \mathbf{e}_{i_{k}}:=S\left(\mathbf{e}_{i_{1}} \otimes \cdots \otimes \mathbf{e}_{i_{k}}\right)=\frac{1}{k !} \sum_{\sigma \in \mathfrak{S}_{k}} \mathbf{e}_{i_{\sigma(1)}} \otimes \cdots \otimes \mathbf{e}_{i_{\sigma(k)}} .
$$

Then since $S \sigma=S$, the term $\mathbf{e}_{i_{1}} \cdots \mathbf{e}_{i_{k}}$ depends only on the number of times each $\mathbf{e}_{i}$ enters this product and we may write

$$
\mathbf{e}_{i_{1}} \cdots \mathbf{e}_{i_{k}}=\mathbf{e}_{1}^{p_{1}} \cdots \mathbf{e}_{n}^{p_{n}}
$$

where $p_{i}$ is the multiplicity (which may be 0 ) of occurrence of $\mathbf{e}_{i}$ in $\mathbf{e}_{i_{1}} \cdots \mathbf{e}_{i_{k}}$. Note that $p_{1}, \ldots, p_{n}$ are nonnegative integers satisfying $p_{1}+\cdots+p_{n}=k$.

Proposition 3.4. Let $\left\{\mathbf{e}_{1}, \ldots, \mathbf{e}_{n}\right\}$ be a basis of $\mathbb{C}^{n}$. Then

$$
\left\{S\left(\mathbf{e}_{i_{1}} \otimes \cdots \otimes \mathbf{e}_{i_{k}}\right) \mid 1 \leq i_{1} \leq \cdots \leq i_{k} \leq n\right\}
$$


or, explicitly,

$$
\left\{\frac{1}{k !} \sum_{\sigma \in \mathfrak{S}_{k}} \mathbf{e}_{i_{\sigma(1)}} \otimes \cdots \otimes \mathbf{e}_{i_{\sigma(k)}} \mid 1 \leq i_{1} \leq \cdots \leq i_{k} \leq n\right\},
$$

is a basis of $\mathrm{S}^{k}\left(\mathbb{C}^{n}\right)$. Furthermore,

$$
\operatorname{dim}_{\mathbb{C}} S^{k}\left(\mathbb{C}^{n}\right)=\left(\begin{array}{c}
n+k-1 \\
k
\end{array}\right) .
$$

Proof. Since $\mathcal{B}=\left\{\mathbf{e}_{i_{1}} \otimes \cdots \otimes \mathbf{e}_{i_{k}} \mid 1 \leq i_{1} \leq n, \ldots, 1 \leq i_{k} \leq n\right\}$ is a basis for $\mathrm{T}^{k}\left(\mathbb{C}^{n}\right)$ and since $S$ maps $\mathrm{T}^{k}\left(\mathbb{C}^{n}\right)$ onto $\mathrm{S}^{k}\left(\mathbb{C}^{n}\right)$, the set

$$
S(\mathcal{B})=\left\{\mathbf{e}_{i_{1}} \cdots \mathbf{e}_{i_{k}} \mid 1 \leq i_{1} \leq \cdots \leq i_{k} \leq n\right\}=\left\{\mathbf{e}_{1}^{p_{1}} \cdots \mathbf{e}_{n}^{p_{n}} \mid p_{1}+\cdots+p_{n}=k\right\}
$$

spans $S^{k}\left(\mathbb{C}^{n}\right)$. Vectors in $S(\mathcal{B})$ are linearly independent: if $\left(p_{1}, \ldots, p_{n}\right) \neq\left(q_{1}, \ldots, q_{n}\right)$, then the tensors $\mathbf{e}_{1}^{p_{1}} \cdots \mathbf{e}_{n}^{p_{n}}$ and $\mathbf{e}_{1}^{q_{1}} \cdots \mathbf{e}_{n}^{q_{n}}$ are respectively linear combinations of two non-intersecting subsets of basis elements of $\mathrm{T}^{k}\left(\mathbb{C}^{n}\right)$. The cardinality of $S(\mathcal{B})$ is precisely number of partitions of $k$ into a sum of $n$ nonnegative integers, i.e. $\left(\begin{array}{c}n+k-1 \\ k\end{array}\right)$.

If we regard $\mathbf{e}_{j}$ in (3.3) as variables (i.e. indeterminates), then every symmetric tensor of order $k$ and dimension $n$ may be uniquely associated with a homogeneous polynomial of degree $k$ in $n$ variables. Recall that these are just polynomials in $n$ variables whose constituting monomials all have the same total degree $k$. Homogeneous polynomials are also called quantics and those of degrees 1,2, and 3 are often called linear forms, quadratic forms, and cubic forms (or just cubics) respectively. From now on, we will use more standard notation for the variables $-x_{j}$ instead of $\mathbf{e}_{j}$. So the monomial on the RHS of (3.3) now becomes $x_{1}^{p_{1}} \cdots x_{n}^{p_{n}}$. To further simplify this notation, we will adopt the following standard multi-index notations:

$$
\mathbf{x}^{p}:=\prod_{k=1}^{n} x_{k}^{p_{k}} \quad \text { and } \quad|\boldsymbol{p}|:=\sum_{k=1}^{n} p_{k},
$$

where $\boldsymbol{p}$ denotes a $k$-vector of nonnegative integers. We will also write $\mathbb{C}\left[x_{1}, \ldots, x_{n}\right]_{k}$ for the set of homogeneous polynomials of degree $k$ in $n$ variables (again a standard notation). Then any symmetric tensor $\llbracket a_{j_{1} \cdots j_{k}} \rrbracket=\llbracket a_{j} \rrbracket \in \mathrm{S}^{k}\left(\mathbb{C}^{n}\right)$ can be associated with a unique homogeneous polynomial $F \in \mathbb{C}\left[x_{1}, \ldots, x_{n}\right]_{k}$ via the expression

$$
F(\mathrm{x})=\sum_{j} a_{j} \mathrm{x}^{p(j)},
$$

where for every $\boldsymbol{j}=\left(j_{1}, \ldots, j_{k}\right)$, one associates bijectively the nonnegative integer vector $\boldsymbol{p}(\boldsymbol{j})=\left(p_{1}(\boldsymbol{j}), \ldots, p_{n}(\boldsymbol{j})\right)$ with $p_{j}(\boldsymbol{j})$ counting the number of times index $j$ appears in $\boldsymbol{j}$ 16, 14]. We have in particular $|\boldsymbol{p}(\boldsymbol{j})|=k$. The converse is true as well, and the correspondence between symmetric tensors and homogeneous polynomials is obviously bijective. Thus

$$
\mathrm{S}^{k}\left(\mathbb{C}^{n}\right) \cong \mathbb{C}\left[x_{1}, \ldots, x_{n}\right]_{k} .
$$

This justifies the use of the Zariski topology, where the elementary closed subsets are the common zeros of a finite number of homogeneous polynomials 48]. Note that for asymmetric tensors, the same association is not possible (although they can still be associated with polynomials via another bijection). As will be subsequently seen, 
this identification of symmetric tensors with homogeneous polynomials will allow us to prove some interesting facts about symmetric tensor rank.

We will now proceed to define a useful 'inner product' on $\mathbb{C}\left[x_{1}, \ldots, x_{n}\right]_{k}$. For any $F, G \in \mathbb{C}\left[x_{1}, \ldots, x_{n}\right]_{k}$ written as

$$
F(\mathbf{x})=\sum_{|\boldsymbol{p}|=k}\left(\begin{array}{c}
k \\
p_{1}, \ldots, p_{n}
\end{array}\right) a_{\boldsymbol{p}} \mathbf{x}^{\boldsymbol{p}}, \quad G(\mathbf{x})=\sum_{|\boldsymbol{p}|=k}\left(\begin{array}{c}
k \\
p_{1}, \ldots, p_{n}
\end{array}\right) b_{\boldsymbol{p}} \mathbf{x}^{\boldsymbol{p}},
$$

we let

$$
\langle F, G\rangle:=\sum_{|\boldsymbol{p}|=k}\left(\begin{array}{c}
k \\
p_{1}, \ldots, p_{n}
\end{array}\right) a_{\boldsymbol{p}} b_{\boldsymbol{p}}=\sum_{p_{1}+\cdots+p_{n}=k} \frac{k !}{p_{1} ! \cdots p_{n} !} a_{p_{1} \cdots p_{n}} b_{p_{1} \cdots p_{n}} .
$$

Note that $\langle\cdot, \cdot\rangle$ cannot be an inner product in the usual sense since $\langle F, F\rangle$ is in general complex valued (recall that for an inner product, we will need $\langle F, F\rangle \geq 0$ for all $F$ ). However, we will show that it is a non-degenerate symmetric bilinear form.

Lemma 3.5. The bilinear form $\langle\cdot, \cdot\rangle: \mathbb{C}\left[x_{1}, \ldots, x_{n}\right]_{k} \times \mathbb{C}\left[x_{1}, \ldots, x_{n}\right]_{k} \rightarrow \mathbb{C}$ defined above is symmetric and non-degenerate. In other words, $\langle F, G\rangle=\langle G, F\rangle$ for every $F, G \in \mathbb{C}\left[x_{1}, \ldots, x_{n}\right]_{k}$; and if $\langle F, G\rangle=0$ for all $G \in \mathbb{C}\left[x_{1}, \ldots, x_{n}\right]_{k}$, then $F \equiv 0$.

Proof. The bilinearity and symmetry is immediate from definition. Suppose $\langle F, G\rangle=0$ for all $G \in \mathbb{C}\left[x_{1}, \ldots, x_{n}\right]_{k}$. Choose $G$ to be the monomials

$$
G_{\boldsymbol{p}}(\mathbf{x})=\left(\begin{array}{c}
k \\
p_{1}, \ldots, p_{n}
\end{array}\right) \mathbf{x}^{p}
$$

where $|\boldsymbol{p}|=k$ and we see immediately that

$$
a_{\boldsymbol{p}}=\left\langle F, G_{\boldsymbol{p}}\right\rangle=0
$$

Thus $F \equiv 0$.

In the special case where $G$ is the $k$ th power of a linear form, we have the following lemma. The main interest in introducing this inner product lies precisely in establishing this lemma.

Lemma 3.6. Let $G=\left(\beta_{1} x_{1}+\cdots+\beta_{n} x_{n}\right)^{k}$. Then for any $F \in \mathbb{C}\left[x_{1}, \ldots, x_{n}\right]_{k}$, we have

$$
\langle F, G\rangle=F\left(\beta_{1}, \ldots, \beta_{n}\right)
$$

i.e. $F$ evaluated at $\left(\beta_{1}, \ldots, \beta_{n}\right) \in \mathbb{C}^{n}$.

Proof. Let $b_{\boldsymbol{p}}=\beta_{1}^{p_{1}} \cdots \beta_{n}^{p_{n}}$ for all $\boldsymbol{p}=\left(p_{1}, \ldots, p_{n}\right)$ such that $|\boldsymbol{p}|=k$. The multinomial expansion then yields

$$
\left(\beta_{1} x_{1}+\cdots+\beta_{n} x_{n}\right)^{k}=\sum_{|\boldsymbol{p}|=k}\left(\begin{array}{c}
k \\
p_{1}, \ldots, p_{n}
\end{array}\right) b_{\boldsymbol{p}} \mathbf{x}^{\boldsymbol{p}}
$$

For any $F(\mathbf{x})=\sum_{|\boldsymbol{p}|=k}\left(\begin{array}{c}k \\ p_{1}, \ldots, p_{n}\end{array}\right) a_{\boldsymbol{p}} \mathbf{x}^{\boldsymbol{p}}$

$$
F\left(\beta_{1}, \ldots, \beta_{n}\right)=\sum_{|\boldsymbol{p}|=k}\left(\begin{array}{c}
k \\
p_{1}, \ldots, p_{n}
\end{array}\right) a_{\boldsymbol{p}} b_{\boldsymbol{p}}=\langle F, G\rangle
$$

as required. 
3.2. Equivalence with usual definition. As mentioned earlier, we will show that a tensor is symmetric in the sense of Definition 3.2 if and only if its corresponding array is symmetric in the sense of Definition 3.1.

Proposition 3.7. Let $A \in \mathrm{T}^{k}\left(\mathbb{C}^{n}\right)$ and $\llbracket a_{j_{1} \cdots j_{k}} \rrbracket \in \mathbb{C}^{n \times \cdots \times n}$ be its corresponding $k$-array. Then

$$
\sigma(A)=A
$$

for all permutations $\sigma \in \mathfrak{S}_{k}$ if and only if

$$
a_{i_{\sigma(1)} \cdots i_{\sigma(k)}}=a_{i_{1} \cdots i_{k}}, \quad i_{1}, \ldots, i_{k} \in\{1, \ldots, n\},
$$

for all permutations $\sigma \in \mathfrak{S}_{k}$.

Proof. Suppose $\llbracket a_{i_{1} \cdots i_{k}} \rrbracket \in \mathbb{C}^{n \times \cdots \times n}$ is symmetric in the sense of Definition 3.1. Then the corresponding tensor

$$
A=\sum_{i_{1}, \ldots, i_{k}=1}^{n} a_{i_{1} \cdots i_{k}} \mathbf{e}_{i_{1}} \otimes \cdots \otimes \mathbf{e}_{i_{k}},
$$

where $\left\{\mathbf{e}_{1}, \ldots, \mathbf{e}_{n}\right\}$ denotes the canonical basis in $\mathbb{C}^{n}$, satisfies the following:

$$
\begin{array}{rlr}
S(A) & =\sum_{i_{1}, \ldots, i_{k}=1}^{n} a_{i_{1} \cdots i_{k}} S\left(\mathbf{e}_{i_{1}} \otimes \cdots \otimes \mathbf{e}_{i_{k}}\right) & \\
& =\frac{1}{k !} \sum_{i_{1}, \ldots, i_{k}=1}^{n} a_{i_{1} \cdots i_{k}}\left[\sum_{\sigma \in \mathfrak{S}_{k}} \mathbf{e}_{i_{\sigma(1)}} \otimes \cdots \otimes \mathbf{e}_{i_{\sigma(k)}}\right] & \\
& =\frac{1}{k !} \sum_{i_{1}, \ldots, i_{k}=1}^{n}\left[\sum_{\sigma \in \mathfrak{S}_{k}} a_{i_{\sigma(1)} \cdots i_{\sigma(k)}}\right] \mathbf{e}_{i_{1}} \otimes \cdots \otimes \mathbf{e}_{i_{k}} & \\
& =\frac{1}{k !} \sum_{i_{1}, \ldots, i_{k}=1}^{n} k ! a_{i_{1} \cdots i_{k}} \mathbf{e}_{i_{1}} \otimes \cdots \otimes \mathbf{e}_{i_{k}} & \left(\llbracket a_{i_{1} \cdots i_{k}} \rrbracket \text { symmetric }\right) \\
& =A . &
\end{array}
$$

Hence $A$ is a symmetric tensor in the sense of Definition 3.2.

Conversely, let $A \in \mathrm{T}^{k}\left(\mathbb{C}^{n}\right)$ be symmetric in the sense of Definition 3.2 and

$$
A=\sum_{i_{1}, \ldots, i_{k}=1}^{n} a_{i_{1} \cdots i_{k}} \mathbf{e}_{i_{1}} \otimes \cdots \otimes \mathbf{e}_{i_{k}}
$$

be the expression of $A$ with respect to $\left\{\mathbf{e}_{i_{1}} \otimes \cdots \otimes \mathbf{e}_{i_{k}} \mid 1 \leq i_{1}, \ldots i_{k} \leq n\right\}$, the canonical basis of $\mathrm{T}^{k}\left(\mathbb{C}^{n}\right)$. Then

$$
S(A)=A
$$

implies

$\sum_{i_{1}, \ldots, i_{k}=1}^{n}\left[\frac{1}{k !} \sum_{\sigma \in \mathfrak{S}_{k}} a_{i_{\sigma(1)} \cdots i_{\sigma(k)}}\right] \mathbf{e}_{i_{1}} \otimes \cdots \otimes \mathbf{e}_{i_{k}}=\sum_{i_{1}, \ldots, i_{k}=1}^{n} a_{i_{1} \cdots i_{k}} \mathbf{e}_{i_{1}} \otimes \cdots \otimes \mathbf{e}_{i_{k}}$.

Since $\left\{\mathbf{e}_{i_{1}} \otimes \cdots \otimes \mathbf{e}_{i_{k}} \mid 1 \leq i_{1}, \ldots i_{k} \leq n\right\}$ is a linearly independent set, we must have

$$
\frac{1}{k !} \sum_{\sigma \in \mathfrak{S}_{k}} a_{i_{\sigma(1)} \cdots i_{\sigma(k)}}=a_{i_{1} \cdots i_{k}} \quad \text { for all } i_{1}, \ldots, i_{k} \in\{1, \ldots, n\} .
$$


For any given $\tau \in \mathfrak{S}_{k}$, we have

$$
\begin{aligned}
a_{i_{\tau(1)} \cdots i_{\tau(k)}} & =\frac{1}{k !} \sum_{\sigma \in \mathfrak{S}_{k}} a_{i_{\sigma(\tau(1))} \cdots i_{\sigma(\tau(k))}} & & \text { (by }(3.6)) \\
& =\frac{1}{k !} \sum_{\sigma \in \tau \mathfrak{S}_{k}} a_{i_{\sigma(1)} \cdots i_{\sigma(k)}} & & \\
& =\frac{1}{k !} \sum_{\sigma \in \mathfrak{S}_{k}} a_{i_{\sigma(1)} \cdots i_{\sigma(k)}} & & \left(\tau \mathfrak{S}_{k}=\mathfrak{S}_{k} \text { as } \mathfrak{S}_{k}\right. \text { is a group) } \\
& =a_{i_{1} \cdots i_{k}} & & \text { (by }(3.6)) .
\end{aligned}
$$

Since this holds for arbitrary $\tau \in \mathfrak{S}_{k}$, the array $\llbracket a_{i_{1} \cdots i_{k}} \rrbracket$ is symmetric in the sense of Definition 3.1. ㅁ

4. Notions of rank for symmetric tensors. We will discuss two notions of rank for symmetric tensors - the outer product rank (defined for all tensors) and the symmetric outer product rank (defined only for symmetric tensors). We will show that under certain conditions, they are one and the same. However it is not known if they are equal on all symmetric tensors in general.

4.1. Outer product decomposition and rank. Any tensor can always be decomposed (possibly non-uniquely) as:

$$
A=\sum_{i=1}^{r} \mathbf{u}_{i} \otimes \mathbf{v}_{i} \otimes \cdots \otimes \mathbf{w}_{i}
$$

The tensor $\operatorname{rank}, \operatorname{rank}(A)$, is defined as the smallest integer $r$ such that this decomposition holds exactly [29, 30]. Among other properties, note that this outer product decomposition remains valid in a ring, and that an outer product decomposition of a multilinear transform of $A$ equals the multilinear transform of an outer product decomposition of $A$. In other words, if (4.1) is an outer product decomposition of $A$, then

$$
A \bullet_{1} L \bullet_{2} M \bullet_{3} \cdots \bullet_{k} N=\sum_{i=1}^{r} L \mathbf{u}_{i} \otimes M \mathbf{v}_{i} \otimes \cdots \otimes N \mathbf{w}_{i}
$$

is an outer product decomposition of $A \bullet_{1} L \bullet_{2} M \bullet_{3} \cdots \bullet_{k} N$, which may also be written as $(L, M, \ldots, N) \cdot A$. The outer product decomposition has often been regarded synonymously as the data analytic models CANDECOMP [1] and PARAFAC [28] where the decomposition is used to analyze multiway psychometric data.

Definition 4.1. The rank of $A=\llbracket a_{j_{1} \cdots j_{k}} \rrbracket \in \mathbb{C}^{d_{1} \times \cdots \times d_{k}}$ is defined as

$$
\operatorname{rank}(A):=\min \left\{r \mid A=\sum_{i=1}^{r} \mathbf{u}_{i} \otimes \mathbf{v}_{i} \otimes \cdots \otimes \mathbf{w}_{i}\right\} .
$$

If $A=\llbracket a_{j_{1} \cdots j_{k}} \rrbracket \in \mathrm{S}^{k}\left(\mathbb{C}^{n}\right)$, then we may also define the notion of symmetric rank via

$$
\operatorname{rank}_{\mathrm{S}}(A):=\min \left\{s \mid A=\sum_{i=1}^{s} \mathbf{y}_{i} \otimes \cdots \otimes \mathbf{y}_{i}\right\}
$$

Note that over $\mathbb{C}$, the coefficients $\lambda_{i}$ appearing in decomposition (1.1) may be set to 1 ; this is legitimate since any complex number admits a $k$ th root in $\mathbb{C}$. Henceforth, we will adopt the following notation

$$
\mathbf{y}^{\otimes k}:=\overbrace{\mathbf{y} \otimes \cdots \otimes \mathbf{y}}^{k \text { copies }} .
$$

If in (4.1), we have $\mathbf{u}_{i}=\mathbf{v}_{i}=\cdots=\mathbf{w}_{i}$ for every $i$, then we may call it a symmetric outer product decomposition, yielding a symmetric rank, $\operatorname{ranks}_{\mathrm{S}}(A)$. Constraints 
other than full symmetry may be relevant in some application areas, such as partial symmetry as in INDSCAL [11, 57, or positivity/non-negativity [41, 50, 54.

The definition of symmetric rank is not vacuous because of the following result.

Lemma 4.2. Let $A \in \mathrm{S}^{k}\left(\mathbb{C}^{n}\right)$. Then there exist $\mathbf{y}_{1}, \ldots, \mathbf{y}_{s} \in \mathbb{C}^{n}$ such that

$$
A=\sum_{i=1}^{s} \mathbf{y}_{i}^{\otimes k}
$$

Proof. What we actually have to prove, is that the vector space generated by the $k$ th powers of linear forms $L(\mathbf{x})^{k}$ (for all $L \in \mathbb{C}^{n}$ ) is not included in a hyperplane of $\mathrm{S}^{k}\left(\mathbb{C}^{n}\right)$. This is indeed true, because otherwise there would exist a non-zero element of $\mathrm{S}^{k}\left(\mathbb{C}^{n}\right)$ which is orthogonal, under the bilinear form $\langle\cdot, \cdot\rangle$, to all $L(\mathbf{x})^{k}$ for $L \in \mathbb{C}^{n}$. Equivalently, by Lemma 3.6, there exists a non-zero polynomial $q(\mathbf{x})$ of degree $k$ such that $q(L)=0$ for all $L \in \mathbb{C}^{n}$. But this is impossible, since a non-zero polynomial does not vanish identically on $\mathbb{C}^{n}$. $\mathrm{B}$

Lemma 4.2 may be viewed as a particular case of a basic result in algebraic geometry, stating that the linear space generated by points of an algebraic variety that is not included in a hyperplane, i.e. a subspace of codimension 1 , is the whole space 27, 18, 48. For completeness, a proof of our special case is given above. Note that it follows from the proof that

$$
\operatorname{ranks}_{\mathrm{S}}(A) \leq\left(\begin{array}{c}
n+k-1 \\
k
\end{array}\right)
$$

for all $A \in \mathrm{S}^{k}\left(\mathbb{C}^{n}\right)$.

On the other hand, given a symmetric tensor $A$, one can compute its outer product decomposition either in $S^{k}\left(\mathbb{C}^{n}\right)$ or in $\mathrm{T}^{k}\left(\mathbb{C}^{n}\right)$. Since the outer product decomposition in $\mathrm{S}^{k}\left(\mathbb{C}^{n}\right)$ is constrained, it follows that for all $A \in \mathrm{S}^{k}\left(\mathbb{C}^{n}\right)$,

$$
\operatorname{rank}(A) \leq \operatorname{ranks}_{\mathrm{S}}(A)
$$

We will show that equality holds generically when $\operatorname{ranks}_{\mathrm{S}}(A) \leq n$ and when $k$ is sufficiently large with respect to $n$, and always holds when $\operatorname{ranks}_{\mathrm{S}}(A)=1,2$. While we do not know if the equality holds in general, we suspect that this is the case as we are unaware of any counterexample.

4.2. Secant varieties of the Veronese variety. Let us recall here the correspondence between symmetric outer product decompositions and secant varieties of the Veronese variety. By the bijective correspondence between symmetric tensors and homogeneous polynomials established in (3.5), we may discuss this in the context of homogeneous polynomials. The set of homogeneous polynomials that may be written as a $k$ th power of a linear form, $\beta(x)^{k}=\left(\beta_{1} x_{1}+\cdots+\beta_{n} x_{n}\right)^{k}$ for $\boldsymbol{\beta}=\left(\beta_{1}, \ldots, \beta_{n}\right) \in \mathbb{C}^{n}$, is a closed algebraic set. We may consider this construction as a map from $\mathbb{C}^{n}$ to the space of symmetric tensors given by

$$
\begin{aligned}
\nu_{n, k}: \mathbb{C}^{n} & \rightarrow \mathbb{C}\left[x_{1}, \ldots, x_{n}\right]_{k} \cong \mathrm{S}^{k}\left(\mathbb{C}^{n}\right), \\
\boldsymbol{\beta} & \mapsto \beta(x)^{k} .
\end{aligned}
$$

The image $\nu_{n, k}\left(\mathbb{C}^{n}\right)$ is called the Veronese variety and is denoted $\mathcal{V}_{n, k}$ 27, 64. Following this point of view, a symmetric tensor is of symmetric rank 1 if it corresponds to a point on the Veronese variety. A symmetric tensor is of symmetric rank $r$ if it 
is a linear combination of $r$ symmetric tensors of symmetric rank 1 but not a linear combination of $r-1$ or fewer such tensors. In other words, a symmetric tensor is of symmetric rank not more than $r$ if it is in the linear space spanned by $r$ points of the Veronese variety. The closure of the union of all linear spaces spanned by $r$ points of the Veronese variety $\mathcal{V}_{n, k}$ is called ${ }^{1}$ the $(r-1)$ th-secant variety of $\mathcal{V}_{n, k}$. See [27, 64] for examples and general properties of these algebraic sets. In the asymmetric case, a corresponding notion is obtained by considering the Segre variety, i.e. the image of the Segre map defined in Section 2

4.3. Why rank can exceed dimension. We are now in a position to state and prove the following proposition, which is related to a classical result in algebraic geometry stating that $r$ points in $\mathbb{C}^{n}$ form the solution set of polynomial equations of degree $\leq r$ [27, pp. 6]. This implies that we can find a polynomial of degree $\leq r-1$ that vanishes at $r-1$ of the points $L_{i}$ but not at the last one, and hence the independence of polynomials $L_{1}^{r-1}, \ldots, L_{r}^{r-1}$ follows. Since this proposition is important to our discussion in Section (via its corollary below), we give a direct and simple proof below.

Proposition 4.3. Let $L_{1}, \ldots, L_{r} \in \mathbb{C}\left[x_{1}, \ldots, x_{n}\right]_{1}$, i.e. linear forms in $n$ variables. If for all $i \neq j, L_{i}$ is not a scalar multiple of $L_{j}$, then for any $k \geq r-1$, the polynomials $L_{1}^{k}, \ldots, L_{r}^{k}$ are linearly independent in $\mathbb{C}\left[x_{1}, \ldots, x_{n}\right]$.

Proof. Let $k \geq r-1$. Suppose that for some $\lambda_{1}, \ldots, \lambda_{r}, \sum_{i=1}^{r} \lambda_{i} L_{i}^{k}=0$. Hence, by the duality property of Lemma 3.6,

$$
\sum_{i=1}^{r} \lambda_{i}\left\langle F, L_{i}^{k}\right\rangle=\sum_{i=1}^{r} \lambda_{i} F\left(L_{i}\right)=0
$$

for all $F \in \mathbb{C}\left[x_{1}, \ldots, x_{n}\right]_{k}$. Let us prove that we can find a homogeneous polynomial $F$ of degree $k$ that vanishes at $L_{1}, \ldots, L_{r-1}$ and not at $L_{r}$.

Consider a homogeneous polynomial $F$ of degree $k \geq r-1$ that is a multiple of the product of $r-1$ linear forms $H_{i}$ vanishing at $L_{i}$ but not at $L_{r}$. We have $F\left(L_{r}\right) \neq 0$ but $F\left(L_{j}\right)=0,1 \leq j \leq r-1$. As a consequence, we must have $\lambda_{r}=0$. By a similar argument, we may show that $\lambda_{i}=0$ for all $i=1, \ldots, r$. It follows that the polynomials $L_{1}^{k}, \ldots, L_{r}^{k}$ are linearly independent.

Notice that the bound $r-1$ on the degree can be reduced by $d$ if a $d$-dimensional linear space containing any $d+1$ of these points does not contain one of the other points [27, pp. 6]red. In this case, we can replace the product of $d+1$ linear forms $H_{i}$ vanishing at $d+1$ points by just 1 linear form vanishing at these $d+1$ points.

COROLlaRY 4.4. Let $\mathbf{v}_{1}, \ldots, \mathbf{v}_{r} \in \mathbb{C}^{n}$ be $r$ pairwise linearly independent vectors. For any integer $k \geq r-1$, the rank-1 symmetric tensors

$$
\mathbf{v}_{1}^{\otimes k}, \ldots, \mathbf{v}_{r}^{\otimes k} \in \mathrm{S}^{k}\left(\mathbb{C}^{n}\right)
$$

are linearly independent.

This corollary extends results of [19, Lemma 2.2, pp. 2] and 33, Appendix]. Note that vectors $\mathbf{v}_{1}, \ldots, \mathbf{v}_{r}$ need not be linearly independent.

EXAMPLE 4.5. Vectors $\mathbf{v}_{1}=(1,0), \mathbf{v}_{2}=(0,1)$, and $\mathbf{v}_{3}=(1,1)$, are pairwise noncollinear but linearly dependent. According to Corollary 4.4, the symmetric tensors

\footnotetext{
${ }^{1}$ This seemingly odd choice, i.e. $r-1$ instead of $r$, is standard 27, 64. The reason being that one wants to be consistent with the usual meaning of a secant, i.e. 1-secant, as a line intersecting two points in the variety.
} 
$\mathbf{v}_{1}^{\otimes k}, \mathbf{v}_{2}^{\otimes k}, \mathbf{v}_{3}^{\otimes k}$ are linearly independent for any $k \geq 2$. Evidently, we see that this holds true for $k=2$ since the matrix below has rank 3 :

$$
\left[\begin{array}{llll}
1 & 0 & 0 & 0 \\
0 & 0 & 0 & 1 \\
1 & 1 & 1 & 1
\end{array}\right] .
$$

4.4. Genericity. Roughly speaking, a property is referred to as typical if it holds true on a non-zero-volume set and generic if is true almost everywhere. Proper definitions will follow later in Section 6. It is important to distinguish between typical and generic properties; for instance, as will be subsequently seen, there can be several typical ranks, but by definition only a single generic rank. We will see that there can be only one typical rank over $\mathbb{C}$, and is thus generic.

Through the bijection (3.4), the symmetric outer product decomposition (4.1) of symmetric tensors can be carried over to quantics, as pointed out in 16 . The bijection allows one to talk indifferently about the symmetric outer product decomposition of order- $k$ symmetric tensors and the decomposition of degree- $k$ quantics into a sum of linear forms raised to the $k$ th power.

For a long time, it was believed that there was no explicit expression for the generic rank. As Reznick pointed out in 47, Clebsh proved that even when the numbers of free parameters are the same on both sides of the symmetric outer product decomposition, the generic rank may not be equal to $\frac{1}{n}\left(\begin{array}{c}n+k-1 \\ k\end{array}\right)$. For example, in the case $(k, n)=(4,3)$, there are $\left(\begin{array}{l}6 \\ 4\end{array}\right)=15$ degrees of freedom but the generic symmetric $\operatorname{rank} R_{\mathrm{S}}(4,3)=6 \neq 5=\frac{1}{3}\left(\begin{array}{l}6 \\ 4\end{array}\right)$. In fact, this holds true over both $\mathbb{R} 47$ and $\mathbb{C}[22$. In Section [7, we will see that the generic rank in $S^{k}\left(\mathbb{C}^{n}\right)$ is now known for any order and dimension due to the ground breaking work of Alexander and Hirschowitz.

The special case of cubics $(k=3)$ is much better known - a complete classification is known since 1964 though a constructive algorithm to compute the symmetric outer product decomposition has only been proposed recently [35]. The simplest case of binary quantics $(n=2)$ has also been known for more than two decades [61, 16, 38 - a result that is used in real world engineering problems 15.

5. Rank and symmetric rank. Let $\bar{R}_{\mathrm{S}}(k, n)$ be the generic symmetric rank and $R_{\mathrm{S}}(k, n)$ be the maximally attainable symmetric rank in the space of symmetric tensors $\mathrm{S}^{k}\left(\mathbb{C}^{n}\right)$. Similarly, let $\bar{R}(k, n)$ be the generic rank and $R(k, n)$ be the maximally attainable rank in the space of order- $k$ dimension- $n$ cubical tensors $\mathrm{T}^{k}\left(\mathbb{C}^{n}\right)$. Since $S^{k}\left(\mathbb{C}^{n}\right)$ is a subspace of $\mathrm{T}^{k}\left(\mathbb{C}^{n}\right)$, generic and maximal ranks (when they exist) are related for every fixed order $k$ and dimension $n$ as follows:

$$
\bar{R}(k, n) \geq \bar{R}_{\mathrm{S}}(k, n), \quad \text { and } \quad R(k, n) \geq R_{\mathrm{S}}(k, n) .
$$

It may seem odd that the inequalities in (5.1) and (4.3) are reversed, but there is no contradiction since the spaces are not the same.

It is then legitimate to ask oneself whether the symmetric rank and the rank are always equal. We show that this holds generically when $\operatorname{rank}_{\mathrm{S}}(A) \leq n$ (Proposition 5.3) or when the order $k$ is sufficiently large relative to the dimension $n$ (Proposition 5.4). This always holds (not just generically) when $\operatorname{rank}_{\mathrm{s}}(A)=1,2$ (Proposition 5.5). We will need some preliminary results in proving these assertions.

Lemma 5.1. Let $\mathbf{y}_{1}, \ldots, \mathbf{y}_{s} \in \mathbb{C}^{n}$ be linearly independent. Then the symmetric tensor defined by

$$
A:=\sum_{i=1}^{s} \mathbf{y}_{i}^{\otimes k}
$$


has $\operatorname{ranks}_{\sin }(A)=s$.

Proof. Suppose $\operatorname{rank}_{\mathrm{S}}(A)=r$. Then there exist $\mathbf{z}_{1}, \ldots, \mathbf{z}_{r} \in \mathbb{C}^{n}$ such that

$$
\sum_{i=1}^{s} \mathbf{y}_{i}^{\otimes k}=A=\sum_{j=1}^{r} \mathbf{z}_{j}^{\otimes k} .
$$

By the linear independence of $\mathbf{y}_{1}, \ldots, \mathbf{y}_{s}$, there exist covectors $\varphi_{1}, \ldots, \varphi_{s} \in\left(\mathbb{C}^{n}\right)^{*}$ that are dual to $\mathbf{y}_{1}, \ldots, \mathbf{y}_{s}$, i.e.

$$
\varphi_{i}\left(\mathbf{y}_{j}\right)= \begin{cases}1 & \text { if } i=j, \\ 0 & \text { if } i \neq j .\end{cases}
$$

Contracting both sides of (5.2) in the first $k-1$ modes with $\varphi_{i}^{\otimes(k-1)} \in \mathrm{S}^{k-1}\left(\left(\mathbb{C}^{n}\right)^{*}\right)$, we get

$$
\mathbf{y}_{i}=\sum_{j=1}^{r} \alpha_{j} \mathbf{z}_{j}
$$

where $\alpha_{j}=\varphi_{i}\left(\mathbf{z}_{j}\right)^{k-1}$. In other words, $\mathbf{y}_{i} \in \operatorname{span}\left\{\mathbf{z}_{1}, \ldots, \mathbf{z}_{r}\right\}$. Since this holds for each $i=1, \ldots, s$, it implies that the $s$ linearly independent vectors $\mathbf{y}_{1}, \ldots, \mathbf{y}_{s}$ are contained in $\operatorname{span}\left\{\mathbf{z}_{1}, \ldots, \mathbf{z}_{r}\right\}$. Hence we must have $r \geq s$. On the other hand, it is clear that $r \leq s$. Thus we must have equality.

Lemma 5.2. Let $s \leq n$. Let $A \in \mathrm{S}^{k}\left(\mathbb{C}^{n}\right)$ with $\operatorname{ranks}_{\mathrm{S}}(A)=s$ and

$$
A=\sum_{i=1}^{s} \mathbf{y}_{i}^{\otimes k}
$$

be a symmetric outer product decomposition of $A$. Then vectors of the set $\left\{\mathbf{y}_{1}, \ldots, \mathbf{y}_{s}\right\}$ are generically linearly independent.

Proof. We will write

$$
\mathcal{Y}_{s}:=\left\{A \in \mathrm{S}^{k}\left(\mathbb{C}^{n}\right) \mid \operatorname{ranks}_{\mathrm{S}}(A) \leq s\right\} \quad \text { and } \quad \mathcal{Z}_{s}:=\left\{A \in \mathrm{S}^{k}\left(\mathbb{C}^{n}\right) \mid \operatorname{ranks}_{\mathrm{S}}(A)=s\right\} \text {. }
$$

Define the map from the space of $n \times s$ matrices to order- $k$ symmetric tensors,

$$
\begin{aligned}
f: \mathbb{C}^{n \times s} & \rightarrow \mathrm{S}^{k}\left(\mathbb{C}^{n}\right), \\
{\left[\mathbf{y}_{1}, \ldots, \mathbf{y}_{s}\right] } & \mapsto \sum_{i=1}^{s} \mathbf{y}_{i}^{\otimes k} .
\end{aligned}
$$

It is clear that $f$ takes $\mathbb{C}^{n \times s}$ onto $\mathcal{Y}_{s}$ (i.e. $f\left(\mathbb{C}^{n \times s}\right)=\mathcal{Y}_{s}$ ). We let $E_{0}$ and $E_{1}$ be the subsets of rank-deficient and full-rank matrices in $\mathbb{C}^{n \times s}$ respectively. So we have the disjoint union

$$
E_{0} \cup E_{1}=\mathbb{C}^{n \times s}, \quad E_{0} \cap E_{1}=\varnothing .
$$

Recall that the full-rank matrices are generic in $\mathbb{C}^{n \times s}$. Recall also that $E_{0}$ is an algebraic set in $\mathbb{C}^{n \times s}$ defined by the vanishing of all $s \times s$ principal minors. By the previous lemma, $f\left(E_{1}\right) \subseteq \mathcal{Z}_{s}$. The set of symmetric tensors,

$$
\sum_{i=1}^{s} \mathbf{y}_{i}^{\otimes k}
$$

in $\mathcal{Z}_{s}$ for which $\left\{\mathbf{y}_{1}, \ldots, \mathbf{y}_{s}\right\}$ is linearly dependent, i.e. $\left[\mathbf{y}_{1}, \ldots, \mathbf{y}_{s}\right]$ is rank deficient, is simply

$$
\mathcal{Z}_{s} \cap f\left(E_{0}\right) .
$$


Since $f$ is a polynomial map and $E_{0}$ is a non-trivial algebraic set, we conclude that $f\left(E_{1}\right)$ is generic in $\mathcal{Z}_{s}$.

Proposition 5.3. Let $A \in \mathrm{S}^{k}\left(\mathbb{C}^{n}\right)$. If $\operatorname{rank}_{\mathrm{S}}(A) \leq n$, then $\operatorname{rank}(A)=\operatorname{rank}_{\mathrm{S}}(A)$ generically.

Proof. Let $r=\operatorname{rank}(A)$ and $s=\operatorname{ranks}_{\mathrm{S}}(A)$. So there exist decompositions

$$
\sum_{j=1}^{r} \mathbf{x}_{j}^{(1)} \otimes \cdots \otimes \mathbf{x}_{j}^{(k)}=A=\sum_{i=1}^{s} \mathbf{y}_{i}^{\otimes k}
$$

By Lemma 5.2, we may assume that for a generic $A \in \mathcal{Z}_{s}$, the vectors $\mathbf{y}_{1}, \ldots, \mathbf{y}_{s}$ are linearly independent. As in the proof of Lemma 5.1, we may find a set of covectors $\varphi_{1}, \ldots, \varphi_{s} \in\left(\mathbb{C}^{n}\right)^{*}$ that are dual to $\mathbf{y}_{1}, \ldots, \mathbf{y}_{s}$, i.e.

$$
\varphi_{i}\left(\mathbf{y}_{j}\right)= \begin{cases}1 & \text { if } i=j, \\ 0 & \text { if } i \neq j .\end{cases}
$$

Contracting both sides of 5.3$)$ in the first $k-1$ modes with $\varphi_{i}^{\otimes(k-1)} \in \mathrm{S}^{k-1}\left(\left(\mathbb{C}^{n}\right)^{*}\right)$, we get

$$
\sum_{j=1}^{r} \alpha_{i j} \mathbf{x}_{j}^{(k)}=\mathbf{y}_{i}
$$

where $\alpha_{i j}=\varphi_{i}\left(\mathbf{x}_{j}^{(1)}\right) \cdots \varphi_{i}\left(\mathbf{x}_{j}^{(k-1)}\right), j=1, \ldots, r$. Since this holds for each $i=$ $1, \ldots, s$, it implies that the $s$ linearly independent vectors $\mathbf{y}_{1}, \ldots, \mathbf{y}_{s}$ are contained in $\operatorname{span}\left\{\mathbf{x}_{1}^{(k)}, \ldots, \mathbf{x}_{r}^{(k)}\right\}$. Hence we must have $r \geq s$. On the other hand, it is clear that $r \leq s$. Thus we must have equality.

We will see below that we could have $\operatorname{rank}(A)=\operatorname{rank}_{\mathrm{S}}(A)$ even when the constituting vectors $\mathbf{y}_{1}, \ldots, \mathbf{y}_{s}$ are not linearly independent. The authors would like to thank David Gross for his help in correcting an error in the original proof.

Proposition 5.4. Let $\mathbf{y}_{1}, \ldots, \mathbf{y}_{s} \in \mathbb{C}^{n}$ be pairwise linearly independent. If $k$ is sufficiently large, then the symmetric tensor defined by

$$
A:=\sum_{i=1}^{s} \mathbf{y}_{i}^{\otimes k}
$$

satisfies $\operatorname{rank}(A)=\operatorname{rank}_{\mathrm{S}}(A)$ generically.

Proof. Let $r=\operatorname{rank}(A)$ and $s=\operatorname{rank}_{\mathrm{S}}(A)$. So there exist decompositions

$$
\sum_{j=1}^{r} \mathbf{x}_{j}^{(1)} \otimes \cdots \otimes \mathbf{x}_{j}^{(k)}=A=\sum_{i=1}^{s} \mathbf{y}_{i}^{\otimes k}
$$

Note that the LHS may be written $\sum_{i=1}^{s} \mathbf{y}_{i}^{\otimes k / 2} \otimes \mathbf{y}_{i}^{\otimes k / 2}$, where we have assumed, without loss of generality, that $k$ is even. By Proposition 4.3, when $k$ is sufficiently large, the order- $(k / 2)$ tensors $\mathbf{y}_{1}^{\otimes k / 2}, \ldots, \mathbf{y}_{s}^{\otimes k / 2}$ are generically linearly independent. Hence we may find functionals $\Phi_{1}, \ldots, \Phi_{s} \in \mathrm{S}^{k / 2}\left(\mathbb{C}^{n}\right)^{*}$ that are dual to $\mathbf{y}_{1}^{\otimes k / 2}, \ldots, \mathbf{y}_{s}^{\otimes k / 2} \in \mathrm{S}^{k / 2}\left(\mathbb{C}^{n}\right)$, i.e.

$$
\Phi_{i}\left(\mathbf{y}_{j}^{\otimes k / 2}\right)= \begin{cases}1 & \text { if } i=j, \\ 0 & \text { if } i \neq j .\end{cases}
$$

Contracting both sides of (5.4) in the first $k / 2$ modes with $\Phi_{i}$, we get

$$
\sum_{j=1}^{r} \alpha_{i j} \mathbf{x}_{j}^{(k / 2+1)} \otimes \cdots \otimes \mathbf{x}_{j}^{(k)}=\mathbf{y}_{i}^{\otimes k / 2},
$$


where $\alpha_{i j}=\Phi_{i}\left(\mathbf{x}_{j}^{(1)} \otimes \cdots \otimes \mathbf{x}_{j}^{(k / 2)}\right), j=1, \ldots, r$. Since this holds for each $i=1, \ldots, s$, it implies that the $s$ linearly independent vectors $\mathbf{y}_{1}^{\otimes k / 2}, \ldots, \mathbf{y}_{s}^{\otimes k / 2}$ are contained in $\operatorname{span}\left\{\mathbf{x}_{1}^{(k / 2+1)} \otimes \cdots \otimes \mathbf{x}_{1}^{(k)}, \ldots, \mathbf{x}_{r}^{(k / 2+1)} \otimes \cdots \otimes \mathbf{x}_{r}^{(k)}\right\}$. Hence we must have $r \geq s$. On the other hand, it is clear that $r \leq s$. Thus we must have equality.

Proposition 5.5. Let $A \in \mathrm{S}^{k}\left(\mathbb{C}^{n}\right)$. If $\operatorname{rank}_{\mathrm{S}}(A)=1$ or 2 , then $\operatorname{rank}(A)=$ $\operatorname{ranks}_{S}(A)$.

Proof. If $\operatorname{ranks}_{\mathrm{S}}(A)=1$, then $\operatorname{rank}(A)=1$ clearly. If $\operatorname{ranks}_{\mathrm{S}}(A)=2$, then

$$
A=\mathbf{y}_{1}^{\otimes k}+\mathbf{y}_{2}^{\otimes k}
$$

for some $\mathbf{y}_{1}, \mathbf{y}_{2} \in \mathbb{C}^{n}$. It is clear that $\mathbf{y}_{1}$ and $\mathbf{y}_{2}$ must be linearly independent or otherwise $\mathbf{y}_{2}=\alpha \mathbf{y}_{1}$ implies that

$$
A=\left(\beta \mathbf{y}_{1}\right)^{\otimes k}
$$

for any $\beta=\left(1+\alpha^{k}\right)^{1 / k}$, contradicting ranks $(A)=2$. It follows from the argument in the proof of Proposition 5.3 with $s=2$ that $\operatorname{rank}(A)=2$.

The following result will be useful later.

Proposition 5.6. Let $\mathbf{v}_{1}$ and $\mathbf{v}_{2}$ be two linearly independent vectors in $\mathbb{C}^{n}$. Then for any $k>1$, the following order- $k$ symmetric tensor:

$$
\begin{aligned}
\mathbf{v}_{1} \otimes \mathbf{v}_{2} \otimes \mathbf{v}_{2} \otimes \cdots \otimes & \mathbf{v}_{2}+\mathbf{v}_{2} \otimes \mathbf{v}_{1} \otimes \mathbf{v}_{2} \otimes \cdots \otimes \mathbf{v}_{2} \\
& +\mathbf{v}_{2} \otimes \mathbf{v}_{2} \otimes \mathbf{v}_{1} \otimes \cdots \otimes \mathbf{v}_{2}+\cdots+\mathbf{v}_{2} \otimes \mathbf{v}_{2} \otimes \mathbf{v}_{2} \otimes \cdots \otimes \mathbf{v}_{1}
\end{aligned}
$$

is of symmetric rank $k$.

Proof. It is not hard to check that the symmetric tensor in (5.5) is associated with the quantic $p\left(z_{1}, z_{2}\right)=z_{1} z_{2}^{k-1}$, up to a constant multiplicative factor (where $z_{1}, z_{2}$ are the first two coordinate variables in $\left.\left(z_{1}, \ldots, z_{n}\right)\right)$.

To prove that this quantic is of symmetric rank $k$, we are going to show that $p\left(z_{1}, z_{2}\right)$ can be decomposed into a sum of powers of linear forms as

$$
p\left(z_{1}, z_{2}\right)=\sum_{i=1}^{k} \lambda_{i}\left(\alpha_{i} z_{1}+\beta_{i} z_{2}\right)^{k} .
$$

There are infinitely many possibilities of choosing coefficients $\left(\alpha_{i}, \beta_{i}\right)$ but we just need to provide one solution. Take $\alpha_{1}=\cdots=\alpha_{r}=1$ and $\beta_{1}, \ldots, \beta_{k}$ distinct such that

$$
\sum_{i=1}^{k} \beta_{i}=0 .
$$

First we express all quantics in terms of the canonical basis scaled by the binomial coefficients:

$$
\left\{z_{1}^{k}, k z_{1}^{k-1} z_{2}, \ldots, k z_{1} z_{2}^{k-1}, z_{2}^{k}\right\}
$$

In this basis, the monomial $k z_{1} z_{2}^{k-1}$ can be represented by a $(k+1)$-dimensional vector containing only one non-zero entry. The quantic $\left(z_{i}+\beta_{i} z_{2}\right)^{k}$ is then represented by the vector

$$
\left[1, \beta_{i}, \beta_{i}^{2}, \ldots, \beta_{i}^{k}\right] \in \mathbb{C}^{k+1}
$$


The existence of coefficients $\lambda_{1}, \ldots, \lambda_{k}$ such that we have the decomposition (5.6) is equivalent to the vanishing of the $(k+1) \times(k+1)$ determinant

$$
\left|\begin{array}{ccccc}
0 & 0 & \cdots & 1 & 0 \\
1 & \beta_{1} & \cdots & \beta_{1}^{k-1} & \beta_{1}^{k} \\
\vdots & \vdots & & \vdots & \vdots \\
1 & \beta_{k} & \cdots & \beta_{k}^{k-1} & \beta_{k}^{k}
\end{array}\right| .
$$

An explicit computation shows that this determinant is $\pm\left(\sum_{i=1}^{k} \beta_{i}\right) V_{k}\left(\beta_{1}, \ldots, \beta_{k}\right)$ where $V_{k}\left(\beta_{1}, \ldots, \beta_{k}\right)$ is the Vandermonde determinant of degree $k-1$ of $\beta_{1}, \ldots, \beta_{k}$. Thus by (5.7), the determinant in (5.8) vanishes.

This proves that the symmetric rank of $z_{1} z_{2}^{k}$ is $\leq k$. Note that the symmetric rank cannot be smaller than $k$ because removing any row of the matrix of (5.8) still yields a matrix of rank $k$, if the $\beta_{i}$ are distinct (see also Proposition 4.3).

This proof is constructive, and gives an algorithm to compute a symmetric outer product decomposition of any binary symmetric tensor of the form (5.5). For example, the reader can check out that the decompositions below may be obtained this way.

EXAMPLE 5.7. The quantics $48 z_{1}^{3} z_{2}$ and $60 z_{1}^{4} z_{2}$ are associated with the symmetric tensors of maximal rank $A_{31}$ and $A_{41}$ respectively. Their symmetric outer product decompositions are given by

$$
\begin{aligned}
& A_{31}=8\left(\mathbf{v}_{1}+\mathbf{v}_{2}\right)^{\otimes 4}-8\left(\mathbf{v}_{1}-\mathbf{v}_{2}\right)^{\otimes 4}-\left(\mathbf{v}_{1}+2 \mathbf{v}_{2}\right)^{\otimes 4}+\left(\mathbf{v}_{1}-2 \mathbf{v}_{2}\right)^{\otimes 4}, \\
& A_{41}=8\left(\mathbf{v}_{1}+\mathbf{v}_{2}\right)^{\otimes 5}-8\left(\mathbf{v}_{1}-\mathbf{v}_{2}\right)^{\otimes 5}-\left(\mathbf{v}_{1}+2 \mathbf{v}_{2}\right)^{\otimes 5}+\left(\mathbf{v}_{1}-2 \mathbf{v}_{2}\right)^{\otimes 5}+48 \mathbf{v}_{1}^{\otimes 5} .
\end{aligned}
$$

The maximal symmetric rank achievable by symmetric tensors of order $k$ and dimension $n=2$ is $k$, i.e. $R_{\mathrm{S}}(k, 2)=k$. One can say that such symmetric tensors lie on a tangent line to the Veronese variety of symmetric rank-1 tensors. In [13], an algorithm has been proposed to decompose binary forms when their rank is not larger than $k / 2$; however, this algorithm would not have found the decompositions above since the symmetric ranks of $A_{31}$ and $A_{41}$ exceed $4 / 2$ and 5/2 respectively.

6. Generic symmetric rank and typical symmetric ranks. For given order and dimension, define the following subsets of symmetric tensors $\mathcal{Y}_{r}:=\left\{A \in \mathrm{S}^{k}\left(\mathbb{C}^{n}\right) \mid\right.$ $\left.\operatorname{rank}_{\mathrm{S}}(A) \leq r\right\}$ and $\mathcal{Z}_{r}:=\left\{A \in \mathrm{S}^{k}\left(\mathbb{C}^{n}\right) \mid \operatorname{rank}_{\mathrm{S}}(A)=r\right\}$. Also, denote the corresponding Zariski closures by $\overline{\mathcal{Y}}_{r}$ and $\overline{\mathcal{Z}}_{r}$ respectively. Recall that the Zariski closure 18 of a set $\mathcal{S}$ is simply the smallest variety containing $\mathcal{S}$. For every $r \in \mathbb{N}$, we clearly have

$$
\mathcal{Y}_{r-1} \cup \mathcal{Z}_{r}=\mathcal{Y}_{r} \quad \text { and } \quad \underbrace{\mathcal{Y}_{1}+\cdots+\mathcal{Y}_{1}}_{r \text { copies }}=\mathcal{Y}_{r} \text {. }
$$

The quantities $\bar{R}_{\mathrm{S}}(k, n)$ and $R_{\mathrm{S}}(k, n)$ may now be formally defined by

$$
\bar{R}_{\mathrm{S}}(k, n):=\min \left\{r \mid \overline{\mathcal{Y}}_{r}=\mathrm{S}^{k}\left(\mathbb{C}^{n}\right)\right\} \quad \text { and } \quad R_{\mathrm{S}}(k, n):=\min \left\{r \mid \mathcal{Y}_{r}=\mathrm{S}^{k}\left(\mathbb{C}^{n}\right)\right\}
$$

By definition, we have $\bar{R}_{\mathrm{S}}(k, n) \leq R_{\mathrm{S}}(k, n)$. We shall prove in this section that a generic symmetric rank always exists in $\mathrm{S}^{k}\left(\mathbb{C}^{n}\right)$, i.e. there is an $r$ such that $\overline{\mathcal{Z}}_{r}=$ $\mathrm{S}^{k}\left(\mathbb{C}^{n}\right)$, and that it is equal to $\bar{R}_{\mathrm{S}}(k, n)$, thus justifying our naming $\bar{R}_{\mathrm{S}}(k, n)$ the generic symmetric rank in Section 5 .

An integer $r$ is not a typical rank if $\mathcal{Z}_{r}$ has zero volume, which means that $\mathcal{Z}_{r}$ is contained in a non-trivial closed set. This definition is somewhat unsatisfactory 
since any mention of 'volume' necessarily involves a choice of measure, which is really irrelevant here. A better definition is as follows.

Definition 6.1. An integer $r$ is a typical rank if $\mathcal{Z}_{r}$ is dense with the Zariski topology, i.e. if $\overline{\mathcal{Z}}_{r}=\mathrm{S}^{k}\left(\mathbb{C}^{n}\right)$. When a typical rank is unique, it may be called generic.

We used the wording "typical" in agreement with previous terminologies [9, 55, 57. Since two dense algebraic sets always intersect over $\mathbb{C}$, there can only be one typical rank over $\mathbb{C}$, and hence is generic. In the remainder of this section, we will write $\bar{R}_{\mathrm{S}}=\bar{R}_{\mathrm{S}}(k, n)$ and $R_{\mathrm{S}}=R_{\mathrm{S}}(k, n)$. We can then prove the following.

Proposition 6.2. The varieties $\overline{\mathcal{Z}}_{r}$ can be ordered by inclusion as follows. If

$$
r_{1}<r_{2}<\bar{R}_{\mathrm{S}}<r_{3} \leq R_{\mathrm{S}}
$$

then

$$
\overline{\mathcal{Z}}_{r_{1}} \varsubsetneqq \overline{\mathcal{Z}}_{r_{2}} \varsubsetneqq \overline{\mathcal{Z}}_{\bar{R}_{\mathrm{S}}} \supsetneq \overline{\mathcal{Z}}_{r_{3}}
$$

Before proving this proposition, we first state two preliminary results. Recall that an algebraic variety is irreducible if it cannot be decomposed as the union of proper subvarieties (cf. 27. pp. 51] and [48, pp. 34]). In algebraic geometry, it is known that the secant varieties of any irreducible variety are irreducible. Nevertheless, we will give a short proof of the following lemma for the sake of completeness.

Lemma 6.3. The sets $\overline{\mathcal{Y}}_{r}, r \geq 1$, are irreducible algebraic varieties.

Proof. For $r \geq 1$, the variety $\overline{\mathcal{Y}}_{r}$ is the closure of the image $\mathcal{Y}_{r}$ of the map

$$
\begin{aligned}
\varphi_{r}: \mathbb{C}^{n \times r} & \rightarrow \mathrm{S}^{k}\left(\mathbb{C}^{n}\right), \\
{\left[\mathbf{u}_{1}, \ldots, \mathbf{u}_{r}\right] } & \mapsto \sum_{i=1}^{r} \mathbf{u}_{i}^{\otimes k} .
\end{aligned}
$$

Consider now two polynomials $f, g$ such that $f g \equiv 0$ on $\overline{\mathcal{Y}}_{r}$. As $\overline{\mathcal{Y}}_{r}$ is the Zariski closure of $\mathcal{Y}_{r}$, this is equivalent to $f g \equiv 0$ on $\mathcal{Y}_{r}$ or

$$
(f g) \circ \varphi_{r}=\left(f \circ \varphi_{r}\right)\left(g \circ \varphi_{r}\right) \equiv 0 .
$$

Thus either $f \equiv 0$ or $g \equiv 0$ on $\mathcal{Y}_{r}$ or equivalently on $\overline{\mathcal{Y}}_{r}$, which proves that $\overline{\mathcal{Y}}_{r}$ is an irreducible variety. For more details on properties of parameterized varieties, see [18]. See also the proof of [51, 9] for third order tensors.

Lemma 6.4. We have $\bar{R}_{\mathrm{S}}=\min \left\{r \mid \overline{\mathcal{Y}}_{r}=\overline{\mathcal{Y}}_{r+1}\right\}$.

Proof. Suppose that there exists $r<\bar{R}_{\mathrm{S}}$ such that $\overline{\mathcal{Y}}_{r}=\overline{\mathcal{Y}}_{r+1}$. Then since $\overline{\mathcal{Y}}_{r} \subseteq \overline{\mathcal{Y}}_{r}+\mathcal{Y}_{1} \subseteq \overline{\mathcal{Y}}_{r+1}=\overline{\mathcal{Y}}_{r}$, we have

$$
\overline{\mathcal{Y}}_{r}=\overline{\mathcal{Y}}_{r}+\mathcal{Y}_{1}=\overline{\mathcal{Y}}_{r}+\mathcal{Y}_{1}+\mathcal{Y}_{1}=\cdots=\overline{\mathcal{Y}}_{r}+\mathcal{Y}_{1}+\cdots+\mathcal{Y}_{1}
$$

As the sum of $R_{\mathrm{S}}$ copies of $\mathcal{Y}_{1}$ is $\mathrm{S}^{k}\left(\mathbb{C}^{n}\right)$, we deduce that $\overline{\mathcal{Y}}_{r}=\mathrm{S}^{k}\left(\mathbb{C}^{n}\right)$ and thus $r \geq \bar{R}_{\mathrm{S}}$, which contradicts our hypothesis. By definition, $\overline{\mathcal{Y}}_{\bar{R}_{\mathrm{S}}}=\overline{\mathcal{Y}}_{\bar{R}_{\mathrm{S}}+1}=\mathrm{S}^{k}\left(\mathbb{C}^{n}\right)$, which proves the lemma. See also the proof of [51] for the asymmetric case.

We are now in a position to prove Proposition 6.2.

Proof of Proposition 6.2. By Lemma 6.4, we deduce that for $r<\bar{R}_{S}$,

$$
\overline{\mathcal{Y}}_{r} \neq \overline{\mathcal{Y}}_{r+1} \text {. }
$$

As $\overline{\mathcal{Y}}_{r}$ is an irreducible variety, we have $\operatorname{dim}\left(\overline{\mathcal{Y}}_{r}\right)<\operatorname{dim}\left(\overline{\mathcal{Y}}_{r+1}\right)$. As $\mathcal{Y}_{r} \cup \mathcal{Z}_{r+1}=\mathcal{Y}_{r+1}$, we deduce that

$$
\overline{\mathcal{Y}}_{r} \cup \overline{\mathcal{Z}}_{r+1}=\overline{\mathcal{Y}}_{r+1}
$$


which implies by the irreducibility of $\overline{\mathcal{Y}}_{r+1}$, that $\overline{\mathcal{Z}}_{r+1}=\overline{\mathcal{Y}}_{r+1}$. Consequently, for $r_{1}<r_{2}<\bar{R}_{S}$, we have

$$
\overline{\mathcal{Z}}_{r_{1}}=\overline{\mathcal{Y}}_{r_{1}} \varsubsetneqq \overline{\mathcal{Z}}_{r_{2}}=\overline{\mathcal{Y}}_{r_{2}} \varsubsetneqq \overline{\mathcal{Z}}_{\bar{R}_{\mathrm{S}}}=\overline{\mathcal{Y}}_{\bar{R}_{\mathrm{S}}}=\mathrm{S}^{k}\left(\mathbb{C}^{n}\right) .
$$

Let us prove now that if $\bar{R}_{\mathrm{S}}<r_{3}$, we have $\overline{\mathcal{Z}}_{r_{3}} \varsubsetneqq \mathrm{S}^{k}\left(\mathbb{C}^{n}\right)$. Suppose that $\overline{\mathcal{Z}}_{r_{3}}=\mathrm{S}^{k}\left(\mathbb{C}^{n}\right)$, then $\mathcal{Z}_{r_{3}}$ is dense in $S^{k}\left(\mathbb{C}^{n}\right)$ as well as $\mathcal{Z}_{\bar{R}_{S}}$ in the Zariski topology. This implies that $\mathcal{Z}_{r_{3}} \cap \mathcal{Z}_{\bar{R}_{5}} \neq \varnothing$, which is false because a tensor cannot have two different ranks. Consequently, we have $\overline{\mathcal{Z}}_{r_{3}} \varsubsetneqq \mathrm{S}^{k}\left(\mathbb{C}^{n}\right)$.

Proposition 6.5. If $1 \leq r \leq R_{\mathrm{S}}$, then $\mathcal{Z}_{r} \neq \overline{\mathcal{Z}}_{r}$.

Proof. Let $r>1$ and $A \in \mathcal{Z}_{r}$. Then by definition of $\mathcal{Y}_{r}$, there exists $A_{0} \in \mathcal{Y}_{r-1}$ and $A_{1} \in \mathcal{Y}_{1}$ such that $A=A_{0}+A_{1}$. As $A_{0} \notin \mathcal{Y}_{r-2}$ (otherwise $A \in \mathcal{Y}_{r-1}$ ) we have $A_{0} \in \mathcal{Z}_{r-1}$. For $\varepsilon \neq 0$, define $A_{\varepsilon}=A_{0}+\varepsilon A_{1}$. We have that $A_{\varepsilon} \in \mathcal{Z}_{r}$, for all $\varepsilon \neq 0$, and $\lim _{\varepsilon \rightarrow 0} A_{\varepsilon}=A_{0}$. This shows that $A_{0} \in \overline{\mathcal{Z}}_{r}-\mathcal{Z}_{r}$, and consequently that $\mathcal{Z}_{r} \neq \overline{\mathcal{Z}}_{r}$.

The above proposition is about the set of symmetric tensors of symmetric rank exactly $r$. But what about those of symmetric rank at most $r$ ? While $\mathcal{Y}_{1}$ is closed as a determinantal variety, we will see from Examples 6.6 and 6.7 as well as Proposition 6.8 that $\mathcal{Y}_{r}$ is generally not closed for $r>1$. This is another major difference from matrices, for which all $\mathcal{Y}_{r}$ are closed sets.

Example 6.6. In dimension $n \geq 2$, and for any order $k>2, \mathcal{Y}_{2}$ is not closed. In fact, take two independent vectors $\mathbf{x}_{i}$ and $\mathbf{x}_{j}$ and define the sequence of symmetric tensors

$$
A_{\varepsilon}(i, j):=\frac{1}{\varepsilon}\left[\left(\mathbf{x}_{i}+\varepsilon \mathbf{x}_{j}\right)^{\otimes k}-\mathbf{x}_{i}^{\otimes k}\right] .
$$

For any $\varepsilon \neq 0, A_{\varepsilon}(i, j)$ is of symmetric rank 2 , but converges in the limit as $\varepsilon \rightarrow 0$ to a symmetric tensor of symmetric rank $k$. In fact, the limiting symmetric tensor is easily seen to be a sum of $k$ rank-1 tensors,

$$
\mathbf{x}_{i} \otimes \mathbf{x}_{j} \otimes \cdots \otimes \mathbf{x}_{j}+\mathbf{x}_{j} \otimes \mathbf{x}_{i} \otimes \cdots \otimes \mathbf{x}_{j}+\cdots+\mathbf{x}_{j} \otimes \mathbf{x}_{j} \otimes \cdots \otimes \mathbf{x}_{i},
$$

which has symmetric rank $k$ by Proposition 5.9.

EXAMPLE 6.7. Let $n=3$ and $k=3$. Then $\mathcal{Y}_{5} \subset \overline{\mathcal{Y}}_{3}$, whereas $3<\bar{R}_{\mathrm{S}}$. In fact, take the symmetric tensor associated with the ternary cubic $p(x, y, z)=x^{2} y-x z^{2}$.

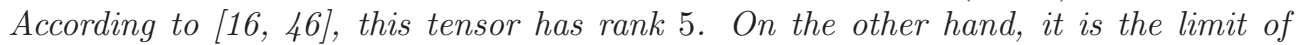
the sequence $p_{\varepsilon}(x, y, z)=x^{2} y-x z^{2}+\varepsilon z^{3}$ as $\varepsilon$ tends to zero. According to a result in [10], the latter polynomial is associated with a rank-3 tensor since the determinant of its Hessian is equal to $8 x^{2}(x-3 \varepsilon z)$ and hence contains two distinct linear forms as long as $\varepsilon \neq 0$.

It is easy to show that this lack of closeness extends in general to $r>\bar{R}_{\mathrm{S}}$ or for $r \leq n$, as stated in the two propositions below.

Proposition 6.8. If $\bar{R}_{\mathrm{S}}<r$, then for all $k>2, \mathcal{Y}_{r} \neq \overline{\mathcal{Y}}_{r}$.

Proof. If $\bar{R}_{\mathrm{S}}<r$, then $\mathcal{Y}_{\bar{R}_{\mathrm{S}}} \varsubsetneqq \mathcal{Y}_{r}$. By the definition of generic symmetric rank, $\overline{\mathcal{Y}}_{\bar{R}_{\mathrm{S}}}=\mathrm{S}^{k}\left(\mathbb{C}^{n}\right)=\overline{\mathcal{Y}}_{r}$. Hence $\mathcal{Y}_{r} \varsubsetneqq \overline{\mathcal{Y}}_{r}=\mathrm{S}^{k}\left(\mathbb{C}^{n}\right)$.

Proposition 6.9. If $1<r \leq n$, then for any $k>2, \mathcal{Y}_{r} \neq \overline{\mathcal{Y}}_{r}$.

Proof. Take $n$ linearly independent vectors $\mathbf{x}_{1}, \ldots, \mathbf{x}_{n}$. Then the symmetric tensors $\mathbf{x}_{1}^{\otimes k}, \ldots, \mathbf{x}_{n}^{\otimes k}$ are linearly independent as well, and $\sum_{i=1}^{r} \mathbf{x}_{i}^{\otimes k}$ is of symmetric rank $r$ for every $r \leq n$ by Lemma 5.1. Now for $r>2$ and any $\varepsilon \neq 0$, define the 
symmetric tensor

$$
A_{\varepsilon}=\frac{1}{\varepsilon}\left[\left(\mathbf{x}_{1}+\varepsilon \mathbf{x}_{2}\right)^{\otimes k}-\mathbf{x}_{1}^{\otimes k}\right]+\sum_{i=3}^{r} \mathbf{x}^{\otimes k} .
$$

$A_{\varepsilon}$ is again of symmetric rank $r$ for every $\varepsilon \neq 0$, but tends to a symmetric rank $r+1$ tensor (see also Section 8.1). For $r=2$, the same reasoning applies with

$$
A_{\varepsilon}=\frac{1}{\varepsilon}\left[\left(\mathbf{x}_{1}+\varepsilon \mathbf{x}_{2}\right)^{\otimes k}-\mathbf{x}_{1}^{\otimes k}\right]
$$

This shows that $\mathcal{Y}_{r}$ is not closed.

Based on these two propositions, we conjecture the stronger statement that for order $k>2$, the set of symmetric tensors of symmetric rank at most $r$ is never closed, even for $r=n+1, \ldots, R_{\mathrm{S}}-1$.

Conjecture 6.10. Assume $k>2$ and $n \geq 2$. Then $\mathcal{Y}_{r} \neq \overline{\mathcal{Y}}_{r}$ for any $r$ such that $1<r<R_{\mathrm{S}}$.

Up to this point, our study has been based on the Zariski topology 44, 18]. However it is useful from a practical point of view to be able to apply these results to other topologies, for example, the Euclidean topology. Since the $\mathcal{Y}_{r}$ 's are parameterized and are thus algebraic constructible sets [48], and since the closure of an algebraic constructible set for the Euclidean topology and the Zariski topology are the same, the results in this paper holds true for many other topologies. We have in particular the following result.

COROLlary 6.11. Let $\mu$ be a measure on Borel subsets of $\mathrm{S}^{k}\left(\mathbb{C}^{n}\right)$ with respect to the Euclidean topology on $\mathrm{S}^{k}\left(\mathbb{C}^{n}\right)$. Let $\bar{R}_{\mathrm{S}}$ be the generic symmetric rank in $\mathrm{S}^{k}\left(\mathbb{C}^{n}\right)$. If $\mu$ is absolutely continuous with respect to the Lebesgue measure on $\mathrm{S}^{k}\left(\mathbb{C}^{n}\right)$, then

$$
\mu\left(\left\{A \in \mathrm{S}^{k}\left(\mathbb{C}^{n}\right) \mid \operatorname{ranks}(A) \neq \bar{R}_{\mathrm{S}}\right\}\right)=0 .
$$

In particular, this corollary tells us that $\mathcal{Z}_{\bar{R}_{S}}$ is also dense in $S^{k}\left(\mathbb{C}^{n}\right)$ with respect to the Euclidean topology. It also tells us that the rank of a tensor whose entries are drawn randomly according to an absolutely continuous distribution (e.g. Gaussian) is $\bar{R}_{\mathrm{S}}$ with probability 1 . This is useful in signal processing for instance, where cumulant tensors are estimated from actual data, and are asymptotically Gaussian distributed [6, 43].

These statements extend previous results [3], and prove that there can be only one subset $\mathcal{Z}_{r}$ of non-empty interior, and that the latter is dense in $\mathrm{S}^{k}\left(\mathbb{C}^{n}\right)$; this result, however, requires that we work over an algebraically closed field such as $\mathbb{C}$.

The results of this section are indeed not generally valid over $\mathbb{R}$. We refer the reader to Section 8 for further discussions concerning the real field.

7. Values of the generic symmetric rank. In practice, it would be useful to be able to compute the symmetric rank of any given symmetric tensor, or at least to know the maximal values of the symmetric rank, given its order and dimensions. Unfortunately, these questions are far from resolved.

The corresponding problem for the generic values of the symmetric rank, however, has seen enormous progress due to the work of Alexander and Hirschowitz described in Section 7.1. In fact, even before their breakthrough, bounds on the generic symmetric rank have been known for decades [3, 46, 47]:

$$
\left\lceil\frac{1}{n}\left(\begin{array}{c}
n+k-1 \\
k
\end{array}\right)\right\rceil \leq \bar{R}_{\mathrm{S}}(k, n) \leq\left(\begin{array}{c}
n+k-2 \\
k-1
\end{array}\right) .
$$


It is known that the lower bound is often accurate but the upper bound is not tight [16. Furthermore, exact results are known in the case of binary quantics $(n=2)$ and ternary cubics $(k=3)$ [22, 16, 47, 35].

7.1. Alexander-Hirschowitz Theorem. It was not until the work [1] of Alexander and Hirschowitz in 1995 that the generic symmetric rank problem was completely settled. Nevertheless, the relevance of their result has remained largely unknown in the applied and computational mathematics communities. One reason is that the connection between our problem and the interpolating polynomials discussed in [1] is not at all well-known in the aforementioned circles. So for the convenience of our readers, we will state the result of Alexander and Hirschowitz in the context of the symmetric outer product decomposition below.

TheORem 7.1 (Alexander-Hirschowitz). For $k>2$, the generic symmetric rank of an order- $k$ symmetric tensor of dimension $n$ over $\mathbb{C}$ is always equal to the lower bound

$$
\bar{R}_{\mathrm{S}}(k, n)=\left\lceil\frac{1}{n}\left(\begin{array}{c}
n+k-1 \\
k
\end{array}\right)\right\rceil
$$

except for the following cases: $(k, n) \in\{(3,5),(4,3),(4,4),(4,5)\}$, where it should be increased by 1 .

This theorem is extremely complicated to prove, and the interested reader should refer to the two papers of Alexander and Hirschowitz [1, 2]. Simplifications to this proof have also been recently proposed in 12 . It is worth noting that these results have been proved in terms of multivariate polynomials and interpolation theory, and not in terms of symmetric tensors. The exception $(k, n)=(4,3)$ has been known since 1860; in fact, Sylvester referred to it as Clebsh Theorem in his work 52. It is not hard to guess the formula in (7.1) by a degrees-of-freedom argument. The difficulty of proving Theorem 7.1 lies in establishing the fact that the four given exceptions to the expected formula (7.1) are the only ones. Table 7.1 below lists a few values of the generic symmetric rank.

\begin{tabular}{|c||ccccccccc|}
\hline$k^{n}$ & 2 & 3 & 4 & 5 & 6 & 7 & 8 & 9 & 10 \\
\hline \hline 3 & 2 & 4 & 5 & $\mathbf{8}$ & 10 & 12 & 15 & 19 & 22 \\
4 & 3 & $\mathbf{6}$ & $\mathbf{1 0}$ & $\mathbf{1 5}$ & 21 & 30 & 42 & 55 & 72 \\
5 & 3 & 7 & 14 & 26 & 42 & 66 & 99 & 143 & 201 \\
6 & 4 & 10 & 21 & 42 & 77 & 132 & 215 & 334 & 501 \\
\hline
\end{tabular}

Values of the generic symmetric rank $\bar{R}_{\mathrm{S}}(k, n)$ for various orders $k$ and dimensions $n$. Values appearing in bold are the exceptions outlined by the Alexander-Hirschowitz Theorem.

\begin{tabular}{|c||ccccccccc|}
\hline$k^{n}$ & 2 & 3 & 4 & 5 & 6 & 7 & 8 & 9 & 10 \\
\hline \hline 3 & 0 & 2 & 0 & $\mathbf{5}$ & 4 & 0 & 0 & 6 & 0 \\
4 & 1 & $\mathbf{3}$ & $\mathbf{5}$ & $\mathbf{5}$ & 0 & 0 & 6 & 0 & 5 \\
5 & 0 & 0 & 0 & 4 & 0 & 0 & 0 & 0 & 8 \\
6 & 1 & 2 & 0 & 0 & 0 & 0 & 4 & 3 & 5 \\
\hline
\end{tabular}

Generic dimension $F(k, n)$ of the fiber of solutions. 
7.2. Uniqueness. Besides the exceptions pointed out in Theorem 7.1, the number of solutions for the symmetric outer product decomposition has to be finite if the rank $r$ is smaller than or equal to $\frac{1}{n}\left(\begin{array}{c}n+k-1 \\ k\end{array}\right)$. This occurs for instance for all cases of degree $k=5$ in Table 7.1, except for $n=5$ and $n=10$. Hence we may deduce the following:

Corollary 7.2. Suppose $(k, n) \notin\{(3,5),(4,3),(4,4),(4,5)\}$. Let $A \in \mathrm{S}^{k}\left(\mathbb{C}^{n}\right)$ be a generic element and let the symmetric outer product decomposition of $A$ be

$$
A=\sum_{i=1}^{\bar{R}_{\mathrm{S}}} \mathbf{v}_{i}^{\otimes k} .
$$

Then (7.2) has a finite number of solutions if and only if

$$
\frac{1}{n}\left(\begin{array}{c}
n+k-1 \\
k
\end{array}\right) \in \mathbb{N} .
$$

Actually, one may easily check the generic dimension of the fiber of solutions by computing the number of remaining free parameters [16]:

$$
F(k, n)=n \bar{R}_{S}(k, n)-\left(\begin{array}{c}
n+k-1 \\
k
\end{array}\right) .
$$

This is summarized in Table 7.2. When the dimension of the fiber is non-zero, there are infinitely many symmetric outer product decompositions.

Our technique is different from the reduction to simplicity proposed by ten Berge et al. [55, 58], but also relies on the calculation of dimensionality.

8. Examples. We will present a few examples to illustrate our discussions in the previous sections.

8.1. Lack of closeness. It has been shown 116, 35 that symmetric tensors of order 3 and dimension 3 have a generic rank $\bar{R}_{\mathrm{S}}(3,3)=4$ and a maximal rank $R_{\mathrm{S}}(3,3)=5$. From the results of Section 6 , this means that only $\mathcal{Z}_{4}$ is dense in $\overline{\mathcal{Y}}_{4}=\overline{\mathcal{Y}}_{5}$, and that $\mathcal{Z}_{3}$ and $\mathcal{Z}_{5}$ are not closed by Proposition 6.5. On the other hand, $\mathcal{Z}_{1}$ is closed.

In order to make this statement even more explicit, let us now define a sequence of symmetric tensors, each of symmetric rank 2 , that converges to a symmetric tensor of symmetric rank 3. This will be a simple demonstration of the lack of closure of $\mathcal{Y}_{r}$ for $r>1$ and $k>2$, already stated in Proposition 6.8. For this purpose, let $\mathbf{x}, \mathbf{y}$ be two non-collinear vectors. Then the following order-3 symmetric tensor is of symmetric rank 2 for any scalar $\varepsilon \neq 0$ :

$$
A_{\varepsilon}=\varepsilon^{2}\left(\mathbf{x}+\varepsilon^{-1} \mathbf{y}\right)^{\otimes 3}+\varepsilon^{2}\left(\mathbf{x}-\varepsilon^{-1} \mathbf{y}\right)^{\otimes 3}
$$

and it converges, as $\varepsilon \rightarrow 0$, to the following symmetric tensor:

$$
A_{0}=2(\mathbf{x} \otimes \mathbf{y} \otimes \mathbf{y}+\mathbf{y} \otimes \mathbf{x} \otimes \mathbf{y}+\mathbf{y} \otimes \mathbf{y} \otimes \mathbf{x}) .
$$

This limiting symmetric tensor is of symmetric rank 3. In fact, one may show 14 that it admits the following symmetric outer product decomposition:

$$
A_{0}=(\mathbf{x}+\mathbf{y})^{\otimes 3}-(\mathbf{x}-\mathbf{y})^{\otimes 3}-2 \mathbf{y}^{\otimes 3} .
$$

Now let $\mathbf{x}_{i}, \mathbf{y}_{i}$ be linearly independent vectors. 
By adding two terms of the form 8.1), a similar example can be given in dimension $n=4$, where we get a sequence of symmetric tensors of symmetric rank 4 converging to a limit of symmetric rank 6 .

We will give two more illustrations of Conjecture 6.10.

EXAMPLE 8.1. If the dimension is $n=3$, we can take three linearly independent vectors, say $\mathbf{x}, \mathbf{y}$, and $\mathbf{z}$. Then the sequence of symmetric tensors $A_{\varepsilon}+\mathbf{z}^{\otimes 3}$ is of symmetric rank 3 and converges towards a symmetric rank-4 tensor.

In dimension 3, it is somewhat more tricky to build a sequence converging towards a symmetric tensor of symmetric rank 5 . Note that 5 is the maximal rank for $k=3$ and $n=3$.

EXAmple 8.2. Consider the sequence below as $\varepsilon$ tends to zero:

$$
\frac{1}{\varepsilon}\left[(\mathbf{x}+\varepsilon \mathbf{y})^{\otimes 3}-\mathbf{x}^{\otimes 3}+(\mathbf{z}+\varepsilon \mathbf{x})^{\otimes 3}-\mathbf{z}^{\otimes 3}\right] .
$$

It converges to the following symmetric tensor, which we expressed as a sum of six (asymmetric) rank-1 terms,

$$
\mathbf{x} \otimes \mathbf{x} \otimes \mathbf{y}+\mathbf{x} \otimes \mathbf{y} \otimes \mathbf{x}+\mathbf{y} \otimes \mathbf{x} \otimes \mathbf{x}+\mathbf{z} \otimes \mathbf{z} \otimes \mathbf{x}+\mathbf{z} \otimes \mathbf{x} \otimes \mathbf{z}+\mathbf{x} \otimes \mathbf{z} \otimes \mathbf{z} .
$$

This has symmetric rank 5 since it can be associated with quantic $x^{2} y+x z^{2}$, which is the sum of (at least) five cubes.

In terms of algebraic geometry, this example admits a simple geometric interpretation. The limiting tensor is the sum of a point in the tangent space to $\mathcal{Y}_{1}$ at $\mathbf{x}^{\otimes 3}$ and a point in the tangent space to $\mathcal{Y}_{1}$ at $\mathbf{z}^{\otimes 3}$.

Note that the same kind of example can be constructed in the asymmetric case:

$$
\mathbf{x}_{1} \otimes \mathbf{x}_{2} \otimes\left(\mathbf{x}_{3}-\varepsilon^{-1} \mathbf{y}_{3}\right)+\left(\mathbf{x}_{1}+\varepsilon \mathbf{y}_{1}\right) \otimes\left(\mathbf{x}_{2}+\varepsilon \mathbf{y}_{2}\right) \otimes \varepsilon^{-1} \mathbf{y}_{3} .
$$

Further discussions of the lack of closeness of $\mathcal{Y}_{r}$ and the ill-posedness of the best rank- $r$ approximation problem in the asymmetric case can be found in [21].

8.2. Symmetric outer product decomposition over the real field. We now turn our attention to real symmetric tensors. We are interested in the symmetric outer product decomposition of $A \in \mathrm{S}^{k}\left(\mathbb{R}^{n}\right)$ over $\mathbb{R}$, i.e.

$$
A=\sum_{i=1}^{r} \lambda_{i} \mathbf{v}_{i} \otimes \mathbf{v}_{i} \otimes \cdots \otimes \mathbf{v}_{i}
$$

where $\lambda_{i} \in \mathbb{R}$ and $\mathbf{v}_{i} \in \mathbb{R}^{n}$ for all $i=1, \ldots, r$. First note that unlike the decomposition over $\mathbb{C}$ in Lemma 4.2 , we can no longer drop the coefficients $\lambda_{1}, \ldots, \lambda_{r}$ in (8.3) since the $k$ th roots of $\lambda_{i}$ may not exist in $\mathbb{R}$.

Since $\mathrm{S}^{k}\left(\mathbb{R}^{n}\right) \subset \mathrm{S}^{k}\left(\mathbb{C}^{n}\right)$, we may regard $A$ as an element of $\mathrm{S}^{k}\left(\mathbb{C}^{n}\right)$ and seek its symmetric outer product decomposition over $\mathbb{C}$. It is easy to see that we will generally need more terms in $(8.3)$ to decompose $A$ over $\mathbb{R}$ than over $\mathbb{C}$ and so

$$
\operatorname{ranks,\mathbb {C}}(A) \leq \operatorname{rank}_{\mathrm{s}, \mathbb{R}}(A) .
$$

This inequality also holds true for the outer product rank of asymmetric tensors. For $k=2$, i.e. matrices, we always have equality in (8.4) but we will see in the examples below that strict inequality can occur when $k>2$.

Example 8.3. Let $A \in \mathrm{S}^{3}\left(\mathbb{R}^{2}\right)$ be defined by

$$
A=\left[\begin{array}{rr|rr}
-1 & 0 & 0 & 1 \\
0 & 1 & 1 & 0
\end{array}\right]
$$


It is of symmetric rank 3 over $\mathbb{R}$ :

$$
A=\frac{1}{2}\left[\begin{array}{l}
1 \\
1
\end{array}\right]^{\otimes 3}+\frac{1}{2}\left[\begin{array}{c}
1 \\
-1
\end{array}\right]^{\otimes 3}-2\left[\begin{array}{l}
1 \\
0
\end{array}\right]^{\otimes 3}
$$

whereas it is of symmetric rank 2 over $\mathbb{C}$ :

$$
A=\frac{\jmath}{2}\left[\begin{array}{c}
-\jmath \\
1
\end{array}\right]^{\otimes 3}-\frac{\jmath}{2}\left[\begin{array}{l}
\jmath \\
1
\end{array}\right]^{\otimes 3}, \text { where } \jmath:=\sqrt{-1} .
$$

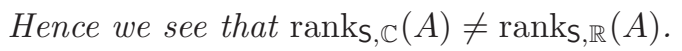

These decompositions may be obtained using the algorithm described in [16], for instance. Alternatively, this tensor is associated with the homogeneous polynomial in two variables $p(x, y)=3 x y^{2}-x^{3}$, which can be decomposed over $\mathbb{R}$ into

$$
p(x, y)=\frac{1}{2}(x+y)^{3}+\frac{1}{2}(x-y)^{3}-2 x^{3} .
$$

In the case of $2 \times 2 \times 2$ symmetric tensors, or equivalently in the case of binary cubics, the symmetric outer product decomposition can always be computed [16]. Hence, the symmetric rank of any symmetric tensor can be calculated, even over $\mathbb{R}$. In this case, it can be shown that the generic symmetric rank over $\mathbb{C}$ is 2 whereas there are two typical symmetric ranks over $\mathbb{R}$, which are 2 and 3

In fact, in the $2 \times 2 \times 2$ case, there are two $2 \times 2$ matrix slices, that we can call $A_{0}$ and $A_{1}$. Since the generic symmetric rank over $\mathbb{C}$ is 2 , the outer product decomposition is obtained via the eigenvalue decomposition of the matrix pencil $\left(A_{0}, A_{1}\right)$, which generically exists and whose eigenvalues are those of $A_{0} A_{1}^{-1}$. By generating (four) independent real Gaussian entries with zero mean and unit variance, it can be easily checked out with a simple computer simulation that one gets real eigenvalues in $52 \%$ of the cases. This means that the real symmetric rank is 3 in $48 \%$ of the remaining cases. This is the simplest example demonstrating that a generic symmetric rank can be lacking over $\mathbb{R}$. So the concept of typical symmetric rank is essential to studying symmetric tensors over $\mathbb{R}$.

For asymmetric tensors, the same kind of computer simulation would yield (by generating 8 independent real Gaussian entries) typical ranks of 2 and 3, $78 \%$ and $22 \%$ of the time, respectively, leading to the same qualitative conclusions. This procedure is not new [53, pp. 13] and has already been proposed in the past to illustrate the existence of several typical ranks for asymmetric tensors [37, 55]. An interesting result obtained by ten Berge [56] is that $p \times p \times 2$ real asymmetric tensors have typical ranks $\{p, p+1\}$.

The problems pertaining to rank and decompositions of real symmetric tensors have not received as much attention as their complex counterparts. However, a moderate amount of work has been done [37, 47, 55, 58, 57 and we refer the reader to these for further information.

8.3. Open questions. Most of the results that we have presented so far are limited to symmetric tensors over the complex field. The case of general asymmetric tensors is currently being addressed with the same kind of approach. As pointed out earlier, decompositions over the real field are more complicated to handle with algebraic geometric tools. In addition, while the problem of determining the generic symmetric rank has been resolved thanks to the Alexander-Hirschowitz Theorem, the 
maximal symmetric rank is known only for particular values of order and dimensions (e.g. dimension 2); only very rough upper bounds are known for general values. Lastly, the computation of an explicit symmetric outer product decomposition for a symmetric tensor is computationally expensive, and the conditions (dimension, order) under which this can be executed within a polynomial time are not yet clearly known. These are problems that we hope will be addressed in future work, either by ourselves or interested readers.

Acknowledgements. The authors would like to thank the anonymous reviewers for their helpful comments. This work is a result of collaboration initiated in the 2004 Workshop on Tensor Decomposition held at the American Institute of Mathematics, Palo Alto, CA, and continued in the 2005 Workshop on Tensor Decomposition and its Applications held at the Centre International de Rencontres Mathématiques (CIRM), Luminy, France. The work of B. Mourrain and P. Comon has been partially supported by the contract ANR-06-BLAN-0074 "DECOTES". The work of G.H. Golub has been partially supported by the grant CCF 0430617 from the National Science Foundation. The work of L.-H. Lim has been partially supported by the grant DMS 0101364 from the National Science Foundation, and by the Gerald J. Lieberman Fellowship from Stanford University.

\section{REFERENCES}

[1] J. Alexander and A. Hirschowitz, "Polynomial interpolation in several variables," J. Algebraic Geom., 4 (1995), no. 2, pp. 201-222.

[2] J. Alexander and A. Hirschowitz, "La méthode d'Horace éclatée: application à l'interpolation en degré quatre," Invent. Math., 107 (1992), no. 3, pp. 585-602.

[3] M.D. Atkinson and S. Lloyd, "Bounds on the ranks of some 3-tensors," Linear Algebra Appl., 31 (1980), pp. 19-31.

[4] D. Bini, M. Capovani, G. Lotti, and F. Romani, " $O\left(n^{2.7799}\right)$ complexity for $n \times n$ approximate matrix multiplication," Inform. Process. Lett., 8 (1979), no. 5, pp. 234-235.

[5] N. Bourbaki, Algebra I: chapters 1-3, Elements of Mathematics, Springer-Verlag, Berlin, 1998.

[6] D.R. Brillinger, Time series: data analysis and theory, Classics in Applied Mathematics, 36, SIAM, Philadelphia, PA, 2001.

[7] A. Brini, R.Q. Huang, and A.G.B. Teolis, "The umbral symbolic method for supersymmetric tensors," Adv. Math., 96 (1992), no. 2, pp. 123-193.

[8] J.W. Brewer, "Kronecker products and matrix calculus in system theory," IEEE Trans. Circuits and Systems, 25 (1978), no. 9, pp. 772-781.

[9] P. Burgisser, M. Clausen, and M.A. Shokrollahi, Algebraic Complexity Theory, 315, SpringerVerlag, Berlin, 1997.

[10] J.F. Cardoso, "Super-symmetric decomposition of the fourth-order cumulant tensor. Blind identification of more sources than sensors," in Proc. IEEE Internat. Conference Acoust. Speech Signal Process. (ICASSP), Toronto, 1991, pp. 3109-3112.

[11] J.D. Carroll and J.J. Chang, "Analysis of individual differences in multidimensional scaling via n-way generalization of Eckart-Young decomposition," Psychometrika, 35 (1970), no. 3, pp. 283-319.

[12] K. Chandler, "Linear systems of cubics singular at general points of projective space," Compositio Math., 134 (2002), no. 3, pp. 269-282.

[13] G. Comas and M. Seiguer, "On the rank of a binary form," arXiv:math/0112311v1, preprint, 2001.

[14] P. Comon, "Tensor decompositions," in Mathematics in Signal Processing V, J.G. McWhirter and I.K. Proudler, Eds., pp. 1-24. Clarendon Press, Oxford, UK, 2002.

[15] P. Comon, "Blind identification and source separation in $2 \times 3$ under-determined mixtures," IEEE Trans. Signal Process., 52 (2004), no. 1, pp. 11-22.

[16] P. Comon and B. Mourrain, "Decomposition of quantics in sums of powers of linear forms," Signal Process., 53 (1996), no. 2, pp. 93-107.

[17] P. Comon and M. Rajih, "Blind identification of under-determined mixtures based on the characteristic function," Signal Process., 86 (2006), no. 9, pp. 2271-2281. 
[18] D. Cox, J. Little, and D. O'Shea, Using algebraic geometry, Graduate Texts in Mathematics, 185, Springer-Verlag, New York, NY, 1998.

[19] L. de Lathauwer, "A link between canonical decomposition in multilinear algebra and simultaneous matrix diagonalization," preprint, 2006.

[20] L. de Lathauwer, B. de Moor, and J. Vandewalle, "A multilinear singular value decomposition," SIAM J. Matrix Anal. Appl., 21 (2000), no. 4, pp. 1253-1278.

[21] V. de Silva and L.-H. Lim, "Tensor rank and the ill-posedness of the best low-rank approximation problem," to appear in SIAM J. Matrix Anal. Appl.

[22] R. Ehrenborg and G.C. Rota, "Apolarity and canonical forms for homogeneous polynomials," European J. Combin., 14 (1993), no. 3, pp. 157-181.

[23] D. Eisenbud, "Lectures on the geometry of syzygies," with a chapter by J. Sidman, Math. Sci. Res. Inst. Publ., 51, pp. 115-152, Cambridge University Press, Cambridge, 2004.

[24] L. Eldén and B. Savas, "A Newton-Grassmann method for computing the best multilinear rank- $\left(r_{1}, r_{2}, r_{3}\right)$ approximation of a tensor," preprint, 2007.

[25] D.S. Freed, Five lectures on supersymmetry, American Mathematical Society, Providence, RI, 1999.

[26] W. Greub, Multilinear algebra, 2nd Ed., Springer-Verlag, New York, NY 1978.

[27] J. Harris, Algebraic geometry: a first course, Graduate Texts in Mathematics, 133, SpringerVerlag, New York, NY, 1998.

[28] R.A. Harshman, "Foundations of the PARAFAC procedure: Models and conditions for an explanatory multimodal factor analysis," UCLA Working Papers in Phonetics, 16 (1970), pp. 1-84.

[29] F.L. Hitchcock, "The expression of a tensor or a polyadic as a sum of products," J. Math. and Phys., 6 (1927), no. 1, pp. 164-189.

[30] F.L. Hitchcock, "Multiple invariants and generalized rank of a $p$-way matrix or tensor," $J$. Math. and Phys., 7 (1927), no. 1, pp. 39-79.

[31] T.D. Howell, "Global properties of tensor rank," Linear Algebra Appl., 22 (1978), pp. 9-23.

[32] J. JáJá, "An addendum to Kronecker's theory of pencils," SIAM J. Appl. Math., 37 (1979), no. 3 , pp. $700-712$.

[33] A.M. Kagan, Y.V. Linnik, and C.R. Rao, Characterization problems in mathematical statistics, John Wiley and Sons, New York, NY, 1973.

[34] E. Kofidis and P. Regalia, "On the best rank-1 approximation of higher-order supersymmetric tensors," SIAM J. Matrix Anal. Appl., 23 (2001/02), no. 3, pp. 863-884.

[35] I.A. Kogan and M.M. Maza, "Computation of canonical forms for ternary cubics," Proc. Internat. Symposium Symbolic Algebr. Comput. (ISAAC), pp. 151-160, ACM Press, New York, NY, 2002.

[36] J.B. Kruskal, "Three-way arrays: rank and uniqueness of trilinear decompositions, with application to arithmetic complexity and statistics," Linear Algebra Appl., 18 (1977), no. 2, pp. $95-138$.

[37] J.B. Kruskal, "Rank, decomposition, and uniqueness for 3-way and n-way arrays," pp. 7-18, in R. Coppi and S. Bolasco, Eds., Multiway data analysis, North-Holland, Amsterdam, 1989.

[38] J. Kung and G. Rota, "The invariant theory of binary forms," Bull. Amer. Math. Soc., 10 (1984), no. 1, pp. 27-85.

[39] S. Lang, Algebra, Rev. 3rd Ed., Graduate Texts in Mathematics, 211, Springer-Verlag, New York, NY, 2002.

[40] S. Lang, Real and functional analysis, 3rd Ed., Graduate Texts in Mathematics, 142, SpringerVerlag, New York, NY, 1993.

[41] L.-H. Lim, "Optimal solutions to non-negative PARAFAC/multilinear NMF always exist," Workshop on Tensor Decompositions and Applications, Centre International de rencontres Mathématiques, Luminy, France, August 29-September 2, 2005.

[42] M. Marcus, Finite dimensional multilinear algebra, Parts I and II, Series of Monographs and Textbooks in Pure and Applied Mathematics, 23, Marcel Dekker, New York, NY, 1973 and 1975 .

[43] P. McCullagh, Tensor methods in statistics, Chapman and Hall, London, 1987.

[44] D.G. Northcott, Multilinear algebra, Cambridge University Press, Cambridge, UK, 1984.

[45] L. Qi, "Eigenvalues of a real supersymmetric tensor," J. Symbolic Comput., 40 (2005), no. 6, pp. 1302-1324.

[46] B. Reznick, "Sums of powers of complex linear forms," preprint, private correspondence, August 1992.

[47] B. Reznick, "Sums of even powers of real linear forms," Mem. Amer. Math. Soc., 96 (1992), no. 463.

[48] I.R. Shafarevitch, Basic algebraic geometry, Grundlehren der mathematischen Wissenschaften, 
213, Springer-Verlag, Berlin, 1977.

[49] N.D. Sidiropoulos, R. Bro, and G.B. Giannakis, "Parallel factor analysis in sensor array processing," IEEE Trans. Signal Process., 48 (2000), no. 8, pp. 2377-2388.

[50] A. Smilde, R. Bro, and P. Geladi, Multi-way analysis, John Wiley, West Sussex, UK, 2004.

[51] V. Strassen, "Rank and optimal computation of generic tensors," Linear Algebra Appl., 52/53 (1983), pp. 645-685.

[52] J.J. Sylvester, "Sur une extension d'un théorème de Clebsch relatif aux courbes du quatrième degré," C. R. Math. Acad. Sci. Paris, 102 (1886), pp. 1532-1534.

[53] J.M.F. ten Berge, "Partial uniqueness in CAndecomp/Parafac," J. Chemometrics, 18 (2004), no. 1 , pp. $12-16$.

[54] J.M.F. ten Berge, H.A.L. Kiers, W.P. Krijnen, "Computational solutions for the problem of negative saliences and nonsymmetry in INDSCAL," J. Classification, 10 (1993), pp. 115-124.

[55] J.M.F. ten Berge and H.A.L. Kiers, "Simplicity of core arrays in three-way principal component analysis and the typical rank of $p \times q \times 2$ arrays," Linear Algebra Appl., 294 (1999), no. 1-3, pp. 169-179.

[56] J.M.F. ten Berge, "The typical rank of tall three-way arrays," Psychometrika, 65 (2000), no. 4 , pp. $525-532$.

[57] J.M.F. ten Berge, N.D. Sidiropoulos, and R. Rocci, "Typical rank and InDSCAL dimensionality for symmetric three-way arrays of order $I \times 2 \times 2$ or $I \times 3 \times 3$," Linear Algebra Appl., 388 (2004), pp. 363-377.

[58] J.M.F. ten Berge, "Simplicity and typical rank of three-way arrays, with applications to Tucker3 analysis with simple cores," J. Chemometrics, 18 (2004), no. 1, pp. 17-21.

[59] L.R. Tucker, "Some mathematical notes on three-mode factor analysis," Psychometrika, 31 (1966), no. 3, pp. 279-311.

[60] V.S. Varadarajan, Supersymmetry for mathematicians: an introduction, Courant Lecture Notes in Mathematics, 11, American Mathematical Society, Providence, RI, 2004.

[61] D.A. Weinstein, "Canonical forms for symmetric tensors," Linear Algebra Appl., 57 (1984), pp. 271-282.

[62] P. West, Introduction to supersymmetry and supergravity, 2nd Ed., World Scientific, Teaneck, NJ, 1990.

[63] T. Yokonuma, Tensor spaces and exterior algebra, Translations of Mathematical Monographs, 108, AMS, Providence, RI, 1992.

[64] F.L. Zak, Tangents and secants of algebraic varieties, Translations of Mathematical Monographs, 127, AMS, Providence, RI, 1993. 